\title{
Langevin Thermostat for Rigid Body Dynamics
}

\author{
Ruslan L. Davidchack, Richard Handel, and M.V. Tretyakov \\ Department of Mathematics, University of Leicester, Leicester, LE1 7RH, United Kingdom
}

\begin{abstract}
We present a new method for isothermal rigid body simulations using the quaternion representation and Langevin dynamics. It can be combined with the traditional Langevin or gradient (Brownian) dynamics for the translational degrees of freedom to correctly sample the $N V T$ distribution in a simulation of rigid molecules. We propose simple, quasi-symplectic second-order numerical integrators and test their performance on the TIP4P model of water. We also investigate the optimal choice of thermostat parameters.
\end{abstract}

\section{INTRODUCTION}

Classical molecular dynamics simulation of an isolated system naturally samples states from a microcanonical $(N V E)$ ensemble, where the number of particles $N$, volume $V$, and total energy of the system $E$ are held constant. However, in many cases it is desirable to study the system in a more experimentally relevant canonical $(N V T)$ ensemble, where the temperature $T$ is specified instead of $E$. In order to sample from the canonical ensemble, the molecular dynamics equations of motion are modified by introducing the interaction of the system with a "thermostat". There exist a large variety of approaches for introducing such a thermostat, which can be roughly classified into two categories: deterministic and stochastic, depending on whether the resulting equations of motion contain a random component (for a review, see, e.g. Ref. 1).

Among various deterministic approaches, those based on coupling the system to a few external degrees of freedom (e.g. Nosé-Hoover thermostat) have become very popular. Given ergodicity in the molecular system dynamics, such thermostats are proven to generate correct canonical ensemble sampling of the system phase space. However, since the thermostat variables are coupled and control directly only global system quantities (e.g. kinetic energy), such thermostats rely on the efficient energy transfer within the system to achieve equipartition within the canonical distribution, such that the average energy of each degree of freedom within the system is equal to $k_{B} T$. Therefore, in a system where the energy transfer between its different parts is slow (e.g., systems combining fast and slow degrees of freedom), the simple Nosé-Hoover thermostat may have difficulty maintaining the same temperature for the different parts of the system. In this case more complicated thermostats are necessary, for example, Nosé-Hoover chain thermostat, or separate thermostats for different parts of the systems (see, e.g. Ref. 3).

The stochastic approach exploits ergodic stochastic differential equations (SDEs) with the Gibbsian (canonical ensemble) invariant measure. For this purpose, Langevin-type equations or gradient systems with noise can be used (see, e.g. Refs. 6, 8, 12, 14, 16 and references therein). Stochastic thermostats, with their independent thermalization of each degree of freedom, provide direct control of equipartition and thus do not need to rely on the efficient energy transfer within the system.

In order to achieve such a direct thermalization of the system, one needs to be able to apply stochastic thermostats to all types of degrees of freedom. The standard Langevin equations for translational degrees of freedom are well known, while Langevin thermostats for systems with constraints have been proposed quite recently ${ }^{4,5}$. In this paper we introduce Langevin equations for the rigid body dynamics in the quaternion representation and propose effective second-order quasi-symplectic numerical integrators for their simulation. These equations can be coupled either with Langevin or Brownian dynamics for the translational degrees of freedom.

In Section I we recall the Hamiltonian system for rigid body dynamics in the quaternion representation from Ref. 2, based on which we derive Langevin and gradientLangevin thermostats. Second-order (in the weak sense) numerical methods for these stochastic systems are constructed in Section III] We test the thermostats and the proposed numerical integrators on the TIP4P model of water ${ }^{19}$. The results of our numerical experiments are presented in Section [V] In particular, we investigate the optimal choice of thermostat parameters and the discretization error of the numerical methods. A summary of the obtained results is given in Section $\mathrm{V}$.

\section{EQUATIONS OF MOTION}

We consider a system of $n$ rigid three-dimensional molecules described by the center-of-mass coordinates $\mathbf{r}=\left(r^{1 \top}, \ldots, r^{n \mathrm{~T}}\right)^{\top} \in \mathbb{R}^{3 n}, r^{j}=\left(r_{1}^{j}, r_{2}^{j}, r_{3}^{j}\right)^{\top} \in \mathbb{R}^{3}$, and the rotational coordinates in the quaternion representation $\mathbf{q}=\left(q^{1 \mathrm{\top}}, \ldots, q^{n \mathrm{\top}}\right)^{\top} \in \mathbb{R}^{4 n}, q^{j}=\left(q_{0}^{j}, q_{1}^{j}, q_{2}^{j}, q_{3}^{j}\right)^{\top} \in$ $\mathbb{R}^{4}$, such that $\left|q^{j}\right|=1$. We use standard matrix notations, and "T" denotes transpose. Following Ref. 2, we write the system Hamiltonian in the form

$$
H(\mathbf{r}, \mathbf{p}, \mathbf{q}, \boldsymbol{\pi})=\frac{\mathbf{p}^{\top} \mathbf{p}}{2 m}+\sum_{j=1}^{n} \sum_{l=1}^{3} V_{l}\left(q^{j}, \pi^{j}\right)+U(\mathbf{r}, \mathbf{q}),
$$

where $\mathbf{p}=\left(p^{1 \top}, \ldots, p^{n \top}\right)^{\top} \in \mathbb{R}^{3 n}, p^{j}=\left(p_{1}^{j}, p_{2}^{j}, p_{3}^{j}\right)^{\top} \in$ $\mathbb{R}^{3}$, are the center-of-mass momenta conjugate to $\mathbf{r}, \boldsymbol{\pi}=$ $\left(\pi^{1 \mathrm{~T}}, \ldots, \pi^{n \mathrm{~T}}\right)^{\mathrm{\top}} \in \mathbb{R}^{4 n}, \pi^{j}=\left(\pi_{0}^{j}, \pi_{1}^{j}, \pi_{2}^{j}, \pi_{3}^{j}\right)^{\mathrm{\top}} \in \mathbb{R}^{4}$, are the angular momenta conjugate to $\mathbf{q}$, and $U(\mathbf{r}, \mathbf{q})$ is the 
potential interaction energy. The second term represents the rotational kinetic energy of the system with

$$
V_{l}(q, \pi)=\frac{1}{8 I_{l}}\left[\pi^{\top} S_{l} q\right]^{2}, \quad q, \pi \in \mathbb{R}^{4}, \quad l=1,2,3,
$$

where the three constant 4-by-4 matrices $S_{l}$ are such that

$$
\begin{gathered}
S_{1} q=\left(-q_{1}, q_{0}, q_{3},-q_{2}\right)^{\top}, \quad S_{2} q=\left(-q_{2},-q_{3}, q_{0}, q_{1}\right)^{\top}, \\
S_{3} q=\left(-q_{3}, q_{2},-q_{1}, q_{0}\right)^{\top},
\end{gathered}
$$

and $I_{l}$ are the principal moments of inertia of the rigid molecule. The Hamilton equations of motion are

$$
\begin{aligned}
\frac{d \mathbf{r}}{d t} & =\frac{\mathbf{p}}{m}, \quad \frac{d \mathbf{p}}{d t}=-\nabla_{\mathbf{r}} U(\mathbf{r}, \mathbf{q}), \\
\frac{d q^{j}}{d t} & =\sum_{l=1}^{3} \nabla_{\pi^{j}} V_{l}\left(q^{j}, \pi^{j}\right), \\
\frac{d \pi^{j}}{d t} & =-\sum_{l=1}^{3} \nabla_{q^{j}} V_{l}\left(q^{j}, \pi^{j}\right)-\nabla_{q^{j}} U(\mathbf{r}, \mathbf{q}), \\
j & =1, \ldots, n .
\end{aligned}
$$

It is easy to check that if the initial conditions are chosen such that $\left|q^{j}(0)\right|=1$, then the corresponding Hamilton equations of motion ensure that

$$
\left|q^{j}(t)\right|=1, \quad j=1, \ldots, n, \text { for all } t \geq 0 .
$$

In the rest of this section we derive stochastic thermostats for this molecular system, which preserve (4). They take the form of ergodic SDEs with the Gibbsian (canonical ensemble) invariant measure possessing the density

$$
\rho(\mathbf{r}, \mathbf{p}, \mathbf{q}, \boldsymbol{\pi}) \propto \exp (-\beta H(\mathbf{r}, \mathbf{p}, \mathbf{q}, \boldsymbol{\pi})),
$$

where $\beta=1 /\left(k_{B} T\right)>0$ is an inverse temperature.

\section{A. Langevin-type equations}

Consider the Langevin-type equations (in the form of Ito $)^{22}$

$$
\begin{aligned}
d R^{j}= & \frac{P^{j}}{m} d t, \quad R^{j}(0)=r^{j}, \\
d P^{j}= & -\nabla_{r^{j}} U(\mathbf{R}, \mathbf{Q}) d t \\
& -\gamma g\left(P^{j}, R^{j}\right) d t+b\left(R^{j}\right) d w^{j}(t), \quad P^{j}(0)=p^{j}, \\
d Q^{j}= & \sum_{l=1}^{3} \nabla_{\pi^{j}} V_{l}\left(Q^{j}, \Pi^{j}\right) d t, \quad Q^{j}(0)=q^{j}, \quad\left|q^{j}\right|=1, \\
d \Pi^{j}= & -\sum_{l=1}^{3} \nabla_{q^{j}} V_{l}\left(Q^{j}, \Pi^{j}\right) d t-\nabla_{q^{j}} U(\mathbf{R}, \mathbf{Q}) d t \\
- & \Gamma G\left(Q^{j}, \Pi^{j}\right) d t+B\left(Q^{j}, \Pi^{j}\right) d W^{j}(t), \quad \Pi^{j}(0)=\pi^{j}, \\
j= & 1, \ldots, n,
\end{aligned}
$$

where $\gamma \geq 0$ and $\Gamma \geq 0$ with $\gamma \Gamma>0$ are the friction coefficients for the translational and rotational motions, respectively, measured in units of inverse time, which control the strength of coupling of the system to the "heat bath"; $g$ is a 3-dimensional appropriately normalized vector; $G$ is a 4 -dimensional vector, which provides a balance in coupling various rotational degrees of freedom with the "heat bath"; $b$ and $B$ are 3 -by-3 and 4-by-4 matrices, respectively; and $\left(\mathbf{w}^{\top}, \mathbf{W}^{\top}\right)^{\top}=\left(w^{1 \top}, \ldots, w^{n \top}, W^{1}, \ldots, W^{n \top}\right)^{\top}$ is a $(3 n+4 n)$-dimensional standard Wiener process with $w^{j}=\left(w_{1}^{j}, w_{2}^{j}, w_{3}^{j}\right)^{\top}$ and $W^{j}=\left(W_{0}^{j}, W_{1}^{j}, W_{2}^{j}, W_{3}^{j}\right)^{\top}$.

For simplicity, we assumed here that $g, G, b$, and $B$ are the same for all $n$ molecules, although one could choose them depending on the molecule number $j$. The latter can be especially useful for systems consisting of significantly different types of molecules. It is also natural to require that each degree of freedom is thermalized by its own independent noise, and in what follows we assume that the matrices $b$ and $B$ are diagonal. Further, we suppose that the coefficients of (6)-(7) are sufficiently smooth functions and the process $X(t)=$ $\left(\mathbf{R}^{\top}(t), \mathbf{P}^{\top}(t), \mathbf{Q}^{\top}(t), \boldsymbol{\Pi}^{\top}(t)\right)^{\top}$ is ergodic, i.e., there exists a unique invariant measure $\mu$ of $X$ and independently of $x \in \mathbb{R}^{14 n}$ there exists the limit

$$
\lim _{t \rightarrow \infty} E \varphi(X(t ; x))=\int \varphi(x) d \mu(x):=\varphi^{e r g}
$$

for any function $\varphi(x)$ with polynomial growth at infinity (see Refs. 7,8, 16, 18 and references therein). Here $X(t ; x)$ is the solution $X(t)$ of (6)-(17) with the initial condition $X(0)=X(0 ; x)=x$.

It is not difficult to see that the solution of (6)-(7) preserves the property (4), i.e.,

$$
\left|Q^{j}(t)\right|=1, \quad j=1, \ldots, n, \quad \text { for all } t \geq 0
$$

Now we find relations between $\gamma, \Gamma, g, G, b$, and $B$ such that the invariant measure $\mu$ is Gibbsian with the density (5). The density $\rho(\mathbf{r}, \mathbf{p}, \mathbf{q}, \boldsymbol{\pi})$ should satisfy the stationary Fokker-Planck equation:

$$
L^{*} \rho=0
$$

where 


$$
\begin{aligned}
L^{*} \rho & :=\sum_{j=1}^{n}\left\{\sum_{i=1}^{3} \frac{b_{i i}^{2}\left(r^{j}\right)}{2} \frac{\partial^{2} \rho}{\left(\partial p_{i}^{j}\right)^{2}}+\sum_{i=1}^{4} \frac{1}{2} \frac{\partial^{2}}{\left(\partial \pi_{i}^{j}\right)^{2}}\left(B_{i i}^{2}\left(q^{j}, \pi^{j}\right) \rho\right)+\nabla_{p^{j}} \cdot\left[\gamma g\left(p^{j}, r^{j}\right) \rho\right]-\nabla_{q^{j}} \cdot\left(\nabla_{\pi^{j}} \sum_{l=1}^{3} V_{l}\left(q^{j}, \pi^{j}\right) \rho\right)\right. \\
& \left.+\nabla_{\pi^{j}} \cdot\left[\left(\nabla_{q^{j}} \sum_{l=1}^{3} V_{l}\left(q^{j}, \pi^{j}\right)+\nabla_{q^{j}} U(\mathbf{r}, \mathbf{q})+\Gamma G\left(q^{j}, \pi^{j}\right)\right) \rho\right]\right\}-\frac{1}{m} \nabla_{\mathbf{r}} \cdot(\mathbf{p} \rho)+\nabla_{\mathbf{p}} \cdot\left[\nabla_{\mathbf{r}} U(\mathbf{r}, \mathbf{q}) \rho\right] .
\end{aligned}
$$

After some calculations, we get the required relations:

$$
\frac{\beta}{m} \frac{b_{i i}^{2}}{2}\left[\frac{\beta}{m}\left(p_{i}^{j}\right)^{2}-1\right]+\gamma \frac{\partial g_{i}}{\partial p_{i}^{j}}-\gamma \frac{\beta}{m} g_{i} p_{i}^{j}=0
$$

and

$$
\begin{aligned}
\beta \frac{B_{i i}^{2}}{2}\left[\beta\left(\frac{\partial H}{\partial \pi_{i}^{j}}\right)^{2}-\frac{\partial^{2} H}{\left(\partial \pi_{i}^{j}\right)^{2}}\right]+\left(\frac{\partial B_{i i}}{\partial \pi_{i}^{j}}\right)^{2} \\
+B_{i i} \frac{\partial^{2} B_{i i}}{\left(\partial \pi_{i}^{j}\right)^{2}}-\beta B_{i i} \frac{\partial B_{i i}}{\partial \pi_{i}^{j}} \frac{\partial H}{\partial \pi_{i}^{j}} \\
+\Gamma \frac{\partial G_{i}}{\partial \pi_{i}^{j}}-\Gamma \beta G_{i} \frac{\partial H}{\partial \pi_{i}^{j}}=0 .
\end{aligned}
$$

Since numerical methods are usually simpler for systems with additive noise, we limit computational consideration of thermostats in this paper to the case of $b_{i i}$ and $B_{i i}$ both being constant. At the same time, we note that general thermostats (6)-(7) with (11)-(12) may have some beneficial features for certain systems but we leave this question for further study. For constant $b_{i i}$ and $B_{i i}$, the relations (11)-(12) take the form

$$
\gamma g_{i}\left(p^{j}, r^{j}\right)=\frac{\beta}{m} \frac{b_{i i}^{2}}{2} p_{i}^{j} \text { and } \Gamma G_{i}\left(q^{j}, \pi^{j}\right)=\beta \frac{B_{i i}^{2}}{2} \frac{\partial H}{\partial \pi_{i}^{j}} .
$$

In the considered molecular model it is natural to have the same value for all $b_{i i}, i=1,2,3$, and the same value for all $B_{i i}, i=1, \ldots, 4$. Further, taking into account the form of the Hamiltonian (10) and that

$$
\begin{aligned}
\nabla_{\pi} \sum_{l=1}^{3} V_{l}(q, \pi) & =\frac{1}{4} \sum_{l=1}^{3} \frac{1}{I_{l}}\left[\pi^{\top} S_{l} q\right] S_{l} q \\
& =\frac{1}{4} \sum_{l=1}^{3} \frac{1}{I_{l}} S_{l} q\left[S_{l} q\right]^{\top} \pi
\end{aligned}
$$

we can write

$$
G(q, \pi)=J(q) \pi \text { and } B_{i i}^{2}=\frac{2 M \Gamma}{\beta}
$$

with

$$
J(q)=\frac{\sum_{l=1}^{3} \frac{1}{I_{l}} S_{l} q\left[S_{l} q\right]^{\top}}{\sum_{l=1}^{3} \frac{1}{I_{l}}} \text { and } M=\frac{4}{\sum_{l=1}^{3} \frac{1}{I_{l}}} .
$$

Note that $\operatorname{Tr} J(q)=|q|^{2}=1$.
Thus, in this paper under 'Langevin thermostat' we understand the following stochastic system

$$
\begin{aligned}
d R^{j}= & \frac{P^{j}}{m} d t, \quad R^{j}(0)=r^{j}, \\
d P^{j}= & -\nabla_{r^{j}} U(\mathbf{R}, \mathbf{Q}) d t \\
& -\gamma P^{j} d t+\sqrt{\frac{2 m \gamma}{\beta}} d w^{j}(t), \quad P^{j}(0)=p^{j}, \\
d Q^{j}= & \sum_{l=1}^{3} \nabla_{\pi^{j}} V_{l}\left(Q^{j}, \Pi^{j}\right) d t, Q^{j}(0)=q^{j},\left|q^{j}\right|=1,(15) \\
d \Pi^{j}= & -\sum_{l=1}^{3} \nabla_{q^{j}} V_{l}\left(Q^{j}, \Pi^{j}\right) d t-\nabla_{q^{j}} U(\mathbf{R}, \mathbf{Q}) d t \\
& -\Gamma J\left(Q^{j}\right) \Pi^{j} d t+\sqrt{\frac{2 M \Gamma}{\beta}} d W^{j}(t), \quad \Pi^{j}(0)=\pi^{j}, \\
j= & 1, \ldots, n,
\end{aligned}
$$

where $J(q)$ and $M$ are from (13), the rest of the notation is as in (6) -(7). We recall that $\gamma$ and $\Gamma$ are free parameters having the physical meaning of the strength of coupling to the heat bath.

Let us fix a molecule and write the equations for the body-fixed angular velocities $\omega_{x}, \omega_{y}$, and $\omega_{z}$ corresponding to the rotational Langevin subsystem (15). To this end, we recall ${ }^{2}$ that

$$
\omega_{x}=2\left(S_{1} Q\right)^{\top} \dot{Q}, \quad \omega_{y}=2\left(S_{2} Q\right)^{\top} \dot{Q}, \quad \omega_{z}=2\left(S_{3} Q\right)^{\top} \dot{Q} .
$$

Then we obtain

$$
\begin{aligned}
d \omega_{x} & =\left(\frac{\tau_{1}}{I_{1}}+\frac{I_{2}-I_{3}}{I_{1}} \omega_{y} \omega_{z}\right) d t-\frac{M \Gamma}{4 I_{1}} \omega_{x} d t \\
& +\frac{1}{I_{1}} \sqrt{\frac{2 M \Gamma}{\beta}} d \Upsilon_{1}, \\
d \omega_{y} & =\left(\frac{\tau_{2}}{I_{2}}+\frac{I_{3}-I_{1}}{I_{2}} \omega_{x} \omega_{z}\right) d t-\frac{M \Gamma}{4 I_{2}} \omega_{y} d t \\
& +\frac{1}{I_{2}} \sqrt{\frac{2 M \Gamma}{\beta}} d \Upsilon_{2}, \\
d \omega_{z} & =\left(\frac{\tau_{3}}{I_{3}}+\frac{I_{1}-I_{2}}{I_{3}} \omega_{x} \omega_{y}\right) d t-\frac{M \Gamma}{4 I_{3}} \omega_{z} d t \\
& +\frac{1}{I_{3}} \sqrt{\frac{2 M \Gamma}{\beta}} d \Upsilon_{3},
\end{aligned}
$$


where $\tau_{i}$ are the torques, $\tau_{i}=-\frac{1}{2}\left(S_{i} Q\right)^{\top} \nabla_{q} U$, and $d \Upsilon_{i}=$ $\frac{1}{2} \sum_{j=1}^{4}\left(S_{i} Q\right)_{j} d W_{j}$, which can be interpreted as random torques. For $\Gamma=0$, (16) coincide with the equations for the angular velocities in Ref. 2. We also note that due to the form of the Hamiltonian (11) the auxiliary velocity $\omega_{0}$ used in Ref. 2 in the derivation of the Hamiltonian system for rigid-body dynamics is identically equal to zero.

\section{B. A mixture of gradient system and Langevin-type equation}

Another possibility of stochastic thermostating of (3) rests on a mixture of a gradient system for the translational dynamics and Langevin-type equation for the rotational dynamics. We note that according to the density of Gibbsian measure (5) the center-of-mass momenta $\mathbf{P}$ are independent Gaussian random variables and they are independent of the other components of the system, so we can avoid simulating $\mathbf{P}$ via a differential equation.

Consider the 'gradient-Langevin thermostat'

$$
d \mathbf{R}=-\frac{\nu}{m} \nabla_{\mathbf{r}} U(\mathbf{R}, \mathbf{Q}) d t+\sqrt{\frac{2 \nu}{m \beta}} d \mathbf{w}(t), \quad \mathbf{R}(0)=\mathbf{r},
$$

$$
\begin{aligned}
d Q^{j} & =\nabla_{\pi^{j}} \sum_{l=1}^{3} V_{l}\left(Q^{j}, \Pi^{j}\right) d t, \quad Q^{j}(0)=q^{j}, \quad\left|q^{j}\right|=1, \\
d \Pi^{j} & =-\nabla_{q^{j}} \sum_{l=1}^{3} V_{l}\left(Q^{j}, \Pi^{j}\right) d t-\nabla_{q^{j}} U(\mathbf{R}, \mathbf{Q}) d t \\
& -\Gamma J\left(Q^{j}\right) \Pi^{j} d t+\sqrt{\frac{2 M \Gamma}{\beta}} d W^{j}(t), \quad \Pi^{j}(0)=\pi^{j}, \\
j & =1, \ldots, n,
\end{aligned}
$$

where all the notation is as in (14)-(15) and, in particular, $J(q)$ and $M$ are from (13). The invariant measure of (17)-(18) is (5) integrated over $\mathbf{p}$. The property (9) is preserved. The gradient-Langevin thermostat has two free parameters, $\nu>0$ and $\Gamma \geq 0$. The latter is the same as in (14)-(15), while the former, measured in units of time, controls the speed of evolution of the gradient subsystem (17).

It is important to note that the gradient system does not have a natural dynamical time evolution similar to Hamiltonian or Langevin dynamics. This is because changing parameter $\nu$ simply leads to a time renormalization of the gradient subsystem (17). However, when linked with the Langevin dynamics for rotational degrees of freedom, as in (17)-(18), parameter $\nu$ controls the "speed" of evolution of the gradient subsystem relative to the speed of the rotational dynamics.

To check that is the density of the invariant measure for (17)-(18), one needs to consider the Fokker-Planck equation (10) with the following operator:

$$
\begin{aligned}
L^{*} \rho & :=\sum_{j=1}^{n}\left\{\frac{\nu}{m \beta} \sum_{i=1}^{3} \frac{\partial^{2} \rho}{\left(\partial r_{i}^{j}\right)^{2}}+\frac{M \Gamma}{\beta} \sum_{i=1}^{4} \frac{\partial^{2}}{\left(\partial \pi_{i}^{j}\right)^{2}} \rho+\nabla_{\pi^{j}} \cdot\left[\left(\nabla_{q^{j}} \sum_{l=1}^{3} V_{l}\left(q^{j}, \pi^{j}\right)+\nabla_{q^{j}} U(\mathbf{r}, \mathbf{q})+\Gamma J\left(q^{j}\right) \pi^{j}\right) \rho\right]\right. \\
& \left.-\nabla_{q^{j}} \cdot\left(\nabla_{\pi^{j}} \sum_{l=1}^{3} V_{l}\left(q^{j}, \pi^{j}\right) \rho\right)\right\}+\frac{\nu}{m} \nabla_{\mathbf{r}} \cdot\left[\nabla_{\mathbf{r}} U(\mathbf{r}, \mathbf{q}) \rho\right] .
\end{aligned}
$$

Let us remark ${ }^{13}$ that the gradient sub-system (17) can be viewed as an overdamped limit of the Langevin trans- 
lational sub-system (14) for a fixed $\mathbf{Q}$.

\section{NUMERICAL INTEGRATORS}

In this section we consider effective second-order numerical methods for the Langevin thermostat (14)-(15) and the gradient-Langevin thermostat (17)-(18). We first recall the idea of quasi-symplectic integrators for Langevin-type equations introduced in Ref. 10 (see also Refs. 11,12) and also some basic facts from stochastic numerics 11 .

Consider the Langevin equations (14)-(15). Let $D_{0} \in$ $\mathbb{R}^{d}, d=14 n$, be a domain with finite volume. The transformation $x=\left(\mathbf{r}^{\top}, \mathbf{p}^{\top}, \mathbf{q}^{\top}, \boldsymbol{\pi}^{\top}\right)^{\top} \mapsto X(t)=X(t ; x)=$ $\left(\mathbf{R}^{\top}(t ; x), \mathbf{P}^{\top}(t ; x), \mathbf{Q}^{\top}(t ; x), \boldsymbol{\Pi}^{\top}(t ; x)\right)^{\top}$ maps $D_{0}$ into the domain $D_{t}$. The volume $V_{t}$ of the domain $D_{t}$ is equal to

$$
\begin{aligned}
V_{t} & =\int_{D_{t}} d X^{1} \ldots d X^{d} \\
& =\int_{D_{0}}\left|\frac{D\left(X^{1}, \ldots, X^{d}\right)}{D\left(x^{1}, \ldots, x^{d}\right)}\right| d x^{1} \ldots d x^{d} .
\end{aligned}
$$

The Jacobian determinant $\mathbb{J}$ is equal to (see, e.g., Ref. 9 ):

$$
\mathbb{J}=\frac{D\left(X^{1}, \ldots, X^{d}\right)}{D\left(x^{1}, \ldots, x^{d}\right)}=\exp (-n(3 \gamma+\Gamma) \cdot t) .
$$

The system (14)-(15) preserves phase volume when $\gamma=0$ and $\Gamma=0$. If $\gamma \geq 0$ and $\Gamma \geq 0$ with $\gamma \Gamma>0$ then phasevolume contractivity takes place.

If we omit the damping terms, $-\gamma P^{j}$ and $-\Gamma J \Pi^{j}$, in (14)-(15) then the system becomes a Hamiltonian system with additive noise ${ }^{9.11}$, i.e., its phase flow preserves symplectic structure. Under $\gamma=0$ and $\Gamma=0$, (14)-(15) takes the form of the deterministic Hamiltonian system (3).

We say that the method based on a one-step approximation $\bar{X}=\bar{X}(t+h ; t, x), h>0$, is symplectic if $\bar{X}$ preserves symplectic structure ${ }^{9.11}$. It is natural to expect that making use of numerical methods, which are close, in a sense, to symplectic ones, has advantages when applying to stochastic systems close to Hamiltonian ones. In Ref. 10 (see also Ref. 11) numerical methods (they are called quasi-symplectic) for Langevin equations were proposed, which satisfy the two structural conditions:

RL1. The method applied to Langevin equations degenerates to a symplectic method when the Langevin system degenerates to a Hamiltonian one.

RL2. The Jacobian determinant $\overline{\mathbb{J}}=D \bar{X} / D x$ does not depend on $x$.

The requirement RL1 ensures closeness of quasisymplectic integrators to the symplectic ones. As it is always assumed, a method is convergent and, consequently,
$\overline{\mathbb{J}}$ is close to $\mathbb{J}$ at any rate. The requirement RL2 is natural since the Jacobian $\mathbb{J}$ of the original system (14)-(15) does not depend on $x$. RL2 reflects the structural properties of the system which are connected with the law of phase volume contractivity. It is often possible to reach a stronger property consisting in the equality $\overline{\mathbb{J}}=\mathbb{J}$.

We usually consider two types of numerical methods for SDEs: mean-square and weak ${ }^{11}$. Mean-square methods are useful for direct simulation of stochastic trajectories while weak methods are sufficient for evaluation of averages and are simpler than mean-square ones. Therefore, weak methods are most suitable for the purposes of this paper. Let us recall11 that a method $\bar{X}$ is weakly convergent with order $p>0$ if

$$
|E \varphi(\bar{X}(T))-E \varphi(X(T))| \leq C h^{p},
$$

where $h>0$ is a time discretization step and $\varphi$ is a sufficiently smooth function with growth at infinity not faster than polynomial. The constant $C$ does not depend on $h$, it depends on the coefficients of a simulated stochastic system, on $\varphi$, and $T$.

\section{A. Numerical schemes for the Langevin thermostat}

We assume that the system (14)-(15) has to be solved on a time interval $[0, T]$ and for simplicity we use a uniform time discretization with the step $h=T / N$. Using standard ideas of stochastic numerics ${ }^{10,11}$ including splitting techniques and the numerical method from Ref. 2 for the deterministic Hamiltonian system (3), we derive two quasi-symplectic integrators for the Langevin system (14)-(15).

The first integrator (Langevin A) is based on splitting the Langevin system (14)-(15) into the Hamiltonian system with additive noise (i.e., (14)-(15) without the damping terms) and the deterministic system of linear differential equations of the form

$$
\begin{aligned}
\dot{\mathbf{p}} & =-\gamma \mathbf{p} \\
\dot{\pi}^{j} & =-\Gamma J\left(q^{j}\right) \pi^{j}, j=1, \ldots, n .
\end{aligned}
$$

We construct a second-order weak quasi-symplectic integrator for the stochastic Hamiltonian system ${ }^{9.11}$ and appropriately concatenate $\frac{10,11}{1}$ it with the exact solution of (22). The resulting numerical method is given below.

Introduce the mapping $\Psi_{l}(t ; q, \pi):(q, \pi) \mapsto(\mathcal{Q}, \Pi)$ defined by

$$
\begin{aligned}
& \mathcal{Q}=\cos \left(\chi_{l} t\right) q+\sin \left(\chi_{l} t\right) S_{l} q, \\
& \Pi=\cos \left(\chi_{l} t\right) \pi+\sin \left(\chi_{l} t\right) S_{l} \pi,
\end{aligned}
$$

where

$$
\chi_{l}=\frac{1}{4 I_{l}} \pi^{\top} S_{l} q
$$

The first quasi-symplectic scheme for (14)-(15) can be written in the form: 


\section{Langevin A}

$$
\begin{aligned}
& \mathbf{P}_{0}=\mathbf{p}, \quad \mathbf{R}_{0}=\mathbf{r}, \quad \mathbf{Q}_{0}=\mathbf{q}, \quad \boldsymbol{\Pi}_{0}=\boldsymbol{\pi}, \\
& \mathcal{P}_{1, k}=\mathbf{P}_{k} \exp (-\gamma h / 2) \text {, } \\
& \Pi_{1, k}^{j}=\exp \left(-\Gamma J\left(Q_{k}^{j}\right) h / 2\right) \Pi_{k}^{j}, \quad j=1, \ldots, n, \\
& \mathcal{P}_{2, k}=\mathcal{P}_{1, k}-\frac{h}{2} \nabla_{\mathbf{r}} U\left(\mathbf{R}_{k}, \mathbf{Q}_{k}\right)+\frac{\sqrt{h}}{2} \sqrt{\frac{2 m \gamma}{\beta}} \boldsymbol{\xi}_{k} \\
& \Pi_{2, k}^{j}=\Pi_{1, k}^{j}-\frac{h}{2} \nabla_{q^{j}} U\left(\mathbf{R}_{k}, \mathbf{Q}_{k}\right)+\frac{\sqrt{h}}{2} \sqrt{\frac{2 M \Gamma}{\beta}} \eta_{k}^{j} \\
& -\frac{h^{2}}{4} \frac{\Gamma}{\beta} Q_{k}^{j}, \quad j=1, \ldots, n, \\
& \mathbf{R}_{k+1}=\mathbf{R}_{k}+\frac{h}{m} \mathcal{P}_{2, k}, \\
& \left(\mathcal{Q}_{1, k}^{j}, \Pi_{3, k}^{j}\right)=\Psi_{3}\left(h / 2 ; Q_{k}^{j}, \Pi_{2, k}^{j}\right), \\
& \left(\mathcal{Q}_{2, k}^{j}, \Pi_{4, k}^{j}\right)=\Psi_{2}\left(h / 2 ; \mathcal{Q}_{1, k}^{j}, \Pi_{3, k}^{j}\right), \\
& \left(\mathcal{Q}_{3, k}^{j}, \Pi_{5, k}^{j}\right)=\Psi_{1}\left(h ; \mathcal{Q}_{2, k}^{j}, \Pi_{4, k}^{j}\right), \\
& \left(\mathcal{Q}_{4, k}^{j}, \Pi_{6, k}^{j}\right)=\Psi_{2}\left(h / 2 ; \mathcal{Q}_{3, k}^{j}, \Pi_{5, k}^{j}\right), \\
& \left(Q_{k+1}^{j}, \Pi_{7, k}^{j}\right)=\Psi_{3}\left(h / 2 ; \mathcal{Q}_{4, k}^{j}, \Pi_{6, k}^{j}\right), \quad j=1, \ldots, n, \\
& \Pi_{8, k}^{j}=\Pi_{7, k}^{j}-\frac{h}{2} \nabla_{q^{j}} U\left(\mathbf{R}_{k+1}, \mathbf{Q}_{k+1}\right) \\
& +\frac{\sqrt{h}}{2} \sqrt{\frac{2 M \Gamma}{\beta}} \eta_{k}^{j}-\frac{h^{2}}{4} \frac{\Gamma}{\beta} Q_{k+1}^{j}, j=1, \ldots, n, \\
& \mathcal{P}_{3, k}=\mathcal{P}_{2, k}-\frac{h}{2} \nabla_{\mathbf{r}} U\left(\mathbf{R}_{k+1}, \mathbf{Q}_{k+1}\right)+\frac{\sqrt{h}}{2} \sqrt{\frac{2 m \gamma}{\beta}} \boldsymbol{\xi}_{k}, \\
& \mathbf{P}_{k+1}=\mathcal{P}_{3, k} \exp (-\gamma h / 2), \\
& \Pi_{k+1}^{j}=\exp \left(-\Gamma J\left(Q_{k+1}^{j}\right) h / 2\right) \Pi_{8, k}^{j}, \quad j=1, \ldots, n, \\
& k=0, \ldots, N-1 \text {, }
\end{aligned}
$$

and

$$
\frac{\partial}{\partial \pi_{i}^{l}} V_{l}\left(q^{j}, \pi^{j}\right)=\frac{\partial}{\partial q_{i}^{l}} V_{l}\left(q^{j}, \pi^{j}\right)=0 \text { for } j \neq l .
$$

As it is usual in stochastic numerics $\frac{11}{}$, we prove convergence of a numerical method under the global Lipschitz assumption on the coefficients of the stochastic system, which can then be relaxed using the concept of rejecting exploding trajectories 12 .

Analogously to the deterministic case ${ }^{2}$, one can verify that the scheme (24) preserves (91), i.e., $\left|Q_{k}^{j}\right|=1, \quad j=$ $1, \ldots, n$, for all $k$. We summarize the properties of the method (24)-(25) in the following statement.

Proposition 1 The numerical scheme (24)-(25) for (14)-(15) is quasi-symplectic, it preserves the structural property (9), and is of weak order two.

We note that one can choose $\boldsymbol{\xi}_{k}=\left(\xi_{1, k}, \ldots, \xi_{3 n, k}\right)^{\mathrm{T}}$ and $\eta_{k}^{j}=\left(\eta_{1, k}^{j}, \ldots, \eta_{4, k}^{j}\right)^{\top}, j=1, \ldots, n$, so that their components are i.i.d. Gaussian random variables with zero mean and unit variance. In this case the weak order of the scheme remains second as when we use the simple discrete distribution (25). Since simulation of the discrete random variables is cheaper than Gaussian ones, it is preferable to use (25) and it was used in all our experiments in this paper. Let us remark in passing that in the case of Gaussian random variables the above scheme also converges in the mean-square sense $\frac{11}{\underline{11}}$ with order one.

Note that $\exp (-\Gamma J(q) h / 2)$ in (24) is the exponent of a matrix. It can be computed using a standard linear algebra package (such as LAPACK). Since $J(q)$ is a symmetric matrix, LAPACK's dsyev routine can be used to obtain the eigen decomposition

$$
J(q)=T(q) \Lambda_{J}(q) T^{\top}(q),
$$

where $T(q)$ is a matrix whose columns are the eigenvectors of $J(q)$ and

$$
\Lambda_{J}(q)=\operatorname{diag}\left(\lambda_{J, 1}, \ldots, \lambda_{J, 4}\right)
$$

$$
P(\theta=0)=2 / 3, \quad P(\theta= \pm \sqrt{3})=1 / 6 .
$$

It is easy to check ${ }^{10,11}$ that the scheme (24) is quasisymplectic. Moreover, the Jacobian $\overline{\mathbb{J}}$ of the corresponding one-step approximation is exactly equal to the Jacobian $\mathbb{J}$ of the original system (14)-(15).

To prove the second order of weak convergence of (24)(25), we compared the corresponding one-step approximation with the one-step approximation corresponding to the standard second-order weak method for SDEs with additive noise from Ref. 11[p. 113]. The following properties are used in this proof:

$$
2 M \sum_{i=0}^{3} \frac{\partial^{2}}{\partial \pi_{i}^{2}} \sum_{l=1}^{3} \nabla_{q} V_{l}(q, \pi)=4 q,
$$

is a diagonal matrix of the corresponding eigenvalues. Then

$$
\exp (-\Gamma J(q) h / 2)=T(q) \exp \left(-\Gamma \Lambda_{J}(q) h / 2\right) T^{\boldsymbol{\top}}(q),
$$

where

$$
\exp \left(-\Gamma \Lambda_{J}(q) h / 2\right)=\operatorname{diag}\left(\mathrm{e}^{-\Gamma \lambda_{J, 1} h / 2}, \ldots, \mathrm{e}^{-\Gamma \lambda_{J, 4} h / 2}\right) .
$$

Alternatively, the matrix exponent $\exp (-\Gamma J(q) h / 2)$ in (24) can be approximated via the Taylor expansion. To ensure the second-order convergence, it is sufficient to approximate it with accuracy $O\left(h^{3}\right)$ at one step; the scheme will remain quasi-symplectic but the Jacobian $\overline{\mathbb{J}}$ will no longer be equal to $\mathbb{J}$ in (20). 
When the parameters $\gamma$ and $\Gamma$ are large (the strong coupling to the "heat bath" conditions), we propose to use a numerical integrator for the Langevin system (14)(15) based on the following splitting:

$$
\begin{gathered}
d \mathbf{P}_{I}=-\gamma \mathbf{P}_{I} d t+\sqrt{\frac{2 m \gamma}{\beta}} d \mathbf{w}(t), \\
d \Pi_{I}^{j}=-\Gamma J(q) \Pi_{I}^{j} d t+\sqrt{\frac{2 M \Gamma}{\beta}} d W^{j}(t) ; \\
d \mathbf{R}_{I I}=\frac{\mathbf{P}_{I I}}{m} d t \\
d \mathbf{P}_{I I}=-\nabla_{\mathbf{r}} U\left(\mathbf{R}_{I I}, \mathbf{Q}_{I I}\right) d t \\
d Q_{I I}^{j}=\nabla_{\pi^{j}} \sum_{l=1}^{3} V_{l}\left(Q_{I I}^{j}, \Pi_{I I}^{j}\right) d t \\
d \Pi_{I I}^{j}=-\nabla_{q^{j}} U\left(\mathbf{R}_{I I}, \mathbf{Q}_{I I}\right) d t \\
\quad-\nabla_{q^{j}} \sum_{l=1}^{3} V_{l}\left(Q_{I I}^{j}, \Pi_{I I}^{j}\right) d t \\
j=1, \ldots, n .
\end{gathered}
$$

The SDEs (27) have the exact solution:

$$
\begin{aligned}
\mathbf{P}_{I}(t) & =\mathbf{P}_{I}(0) e^{-\gamma t}+\sqrt{\frac{2 m \gamma}{\beta}} \int_{0}^{t} e^{-\gamma(t-s)} d \mathbf{w}(s), \\
\Pi_{I}^{j}(t) & =\exp (-\Gamma J(q) t) \Pi_{I}^{j}(0) \\
& +\sqrt{\frac{2 M \Gamma}{\beta}} \int_{0}^{t} \exp (-\Gamma J(q)(t-s)) d W^{j}(s) .
\end{aligned}
$$

To construct a method based on the splitting (27)-(28), we take half a step of (27) using (29), one step of a symplectic method for (28), and again half a step of (27).

The Ito integral in the expression for $\Pi_{I}^{j}$ in (29) is a four-dimensional Gaussian vector with zero mean and the covariance matrix

$$
\begin{aligned}
C(t ; q) & =\frac{2 M \Gamma}{\beta} \int_{0}^{t} \exp [-2 \Gamma J(q)(t-s)] d s \\
& =\frac{M}{\beta} T(q) \Lambda_{C}(t ; q, \Gamma) T^{\top}(q),
\end{aligned}
$$

where $T(q)$ is as in (26) and

$$
\Lambda_{C}(t ; q, \Gamma)=\operatorname{diag}\left(\lambda_{C, 1}, \ldots, \lambda_{C, 4}\right)
$$

with

$$
\begin{aligned}
\lambda_{C, i}(t ; q, \Gamma) & =\left\{\begin{array}{cc}
2 \Gamma t, & \text { if } \lambda_{J, i}=0 \\
\frac{1-\exp \left(-2 \Gamma \lambda_{J, i}(q) t\right)}{\lambda_{J, i}(q)}, & \text { otherwise. }
\end{array}\right. \\
i & =1, \ldots, 4
\end{aligned}
$$

We note that at least one eigenvalue of $J(q)$ equals zero by definition.
Finally, introduce a $4 \times 4$-dimensional matrix $\sigma(t, q)$ such that

$$
\sigma(t ; q) \sigma^{\top}(t ; q)=C(t ; q) .
$$

Since $C(t ; q)$ is a symmetric matrix, $\sigma(t ; q)$ can be determined as a lower triangular matrix in the Cholesky decomposition of $C(t ; q)$. LAPACK's dpotrf can be used for this purpose.

With the above definitions, we obtain the following quasi-symplectic scheme for (14)-(15):

\section{Langevin B}

$$
\begin{aligned}
& \mathbf{P}_{0}=\mathbf{p}, \quad \mathbf{R}_{0}=\mathbf{r}, \quad \mathbf{Q}_{0}=\mathbf{q}, \quad \Pi_{0}=\pi, \\
& \mathcal{P}_{1, k}=\mathbf{P}_{k} e^{-\gamma h / 2}+\sqrt{\frac{m}{\beta}\left(1-e^{-\gamma h}\right)} \boldsymbol{\xi}_{k} \\
& \Pi_{1, k}^{j}=\exp \left(-\Gamma J\left(Q_{k}^{j}\right) h / 2\right) \Pi_{k}^{j} \\
& +\sigma\left(h / 2 ; Q_{k}^{j}\right) \eta_{k}^{j}, \quad j=1, \ldots, n, \\
& \mathcal{P}_{2, k}=\mathcal{P}_{1, k}-\frac{h}{2} \nabla_{\mathbf{r}} U\left(\mathbf{R}_{k}, \mathbf{Q}_{k}\right), \\
& \Pi_{2, k}^{j}=\Pi_{1, k}^{j}-\frac{h}{2} \nabla_{q^{j}} U\left(\mathbf{R}_{k}, \mathbf{Q}_{k}\right), \quad j=1, \ldots, n, \\
& \mathbf{R}_{k+1}=\mathbf{R}_{k}+\frac{h}{m} \mathcal{P}_{2, k}, \\
& \left(\mathcal{Q}_{1, k}^{j}, \Pi_{3, k}^{j}\right)=\Psi_{3}\left(h / 2 ; Q_{k}^{j}, \Pi_{2, k}^{j}\right), \\
& \left(\mathcal{Q}_{2, k}^{j}, \Pi_{4, k}^{j}\right)=\Psi_{2}\left(h / 2 ; \mathcal{Q}_{1, k}^{j}, \Pi_{3, k}^{j}\right) \\
& \left(\mathcal{Q}_{3, k}^{j}, \Pi_{5, k}^{j}\right)=\Psi_{1}\left(h ; \mathcal{Q}_{2, k}^{j}, \Pi_{4, k}^{j}\right), \\
& \left(\mathcal{Q}_{4, k}^{j}, \Pi_{6, k}^{j}\right)=\Psi_{2}\left(h / 2 ; \mathcal{Q}_{3, k}^{j}, \Pi_{5, k}^{j}\right) \\
& \left(Q_{k+1}^{j}, \Pi_{7, k}^{j}\right)=\Psi_{3}\left(h / 2 ; \mathcal{Q}_{4, k}^{j}, \Pi_{6, k}^{j}\right), \quad j=1, \ldots, n, \\
& \Pi_{8, k}^{j}=\Pi_{7, k}^{j}-\frac{h}{2} \nabla_{q^{j}} U\left(\mathbf{R}_{k+1}, \mathbf{Q}_{k+1}\right), j=1, \ldots, n, \\
& \mathcal{P}_{3, k}=\mathcal{P}_{2, k}-\frac{h}{2} \nabla_{\mathbf{r}} U\left(\mathbf{R}_{k+1}, \mathbf{Q}_{k+1}\right), \\
& \mathbf{P}_{k+1}=\mathcal{P}_{3, k} e^{-\gamma h / 2}+\sqrt{\frac{m}{\beta}\left(1-e^{-\gamma h}\right)} \boldsymbol{\zeta}_{k}, \\
& \Pi_{k+1}^{j}=\exp \left(-\Gamma J\left(Q_{k+1}^{j}\right) h / 2\right) \Pi_{8, k}^{j} \\
& +\sigma\left(h / 2 ; Q_{k+1}^{j}\right) \varsigma_{k}^{j}, \quad j=1, \ldots, n, \\
& k=0, \ldots, N-1 \text {, }
\end{aligned}
$$

where $\boldsymbol{\xi}_{k}=\left(\xi_{1, k}, \ldots, \xi_{3 n, k}\right)^{\top}, \boldsymbol{\zeta}_{k}=\left(\zeta_{1, k}, \ldots, \zeta_{3 n, k}\right)^{\top}$ and $\eta_{k}^{j}=\left(\eta_{1, k}^{j}, \ldots, \eta_{4, k}^{j}\right)^{\mathrm{T}}, \varsigma_{k}^{j}=\left(\varsigma_{1, k}^{j}, \ldots, \varsigma_{4, k}^{j}\right)^{\mathrm{T}}, j=$ $1, \ldots, n$, with their components being i.i.d. with the same law (25).

As in the case of the scheme (24)-(25), the Jacobian $\overline{\mathbb{J}}$ of the one-step approximation corresponding to the integrator (33), (25) is exactly equal to the Jacobian $\mathbb{J}$ of the original system (14)-(15). The following proposition can be proved. 
Proposition 2 The numerical scheme (33), (25) for (14)-(15) is quasi-symplectic, it preserves the structural property (9), and is of weak order two.

We note that if we omit the rotational component in (14)-(15), the scheme (24)-(25) coincides with a secondorder weak quasi-symplectic method from Ref. 10 (see also Refs. 11, 12) and the scheme (33), (25) is close to the one from Ref. 14 (see also Refs. 6, 10,11, 15). Both stochastic integrators (24)-(25) and (33), (25) degenerate to the deterministic scheme from Ref. 2 when $\gamma=0$ and $\Gamma=0$. The scheme (33), (25) is usually preferable when $\gamma$ and/or $\Gamma$ are large (see our experimental results in Section [V] and a discussion in the case of translational Langevin equations in Ref. 15). It is slightly more expensive than (24)-(25) due to the need of generating the additional $7 n$ random variables $\zeta_{i, k}$ and $\varsigma_{k}^{j}$ per step and computing Cholesky factorization. However, for most molecular system of practical interest in computational chemistry and physics, where majority of the computational effort is spent on force calculations, the additional cost is negligible.

\section{B. Numerical scheme for the gradient-Langevin system}

To construct the numerical scheme for the gradientLangevin system (17)-(18), we exploit the Runge-Kutta method of order two for equations with additive noise from Ref. 11[p. 113] to simulate the "gradient" part (17) and the "Langevin" rotational part (18) is approximated in the same way as in (33). The resulting second-order weak scheme has the form

$$
\begin{gathered}
\underline{\text { gradient-Langevin }} \\
\mathbf{R}_{0}=\mathbf{r}, \mathbf{Q}_{0}=\mathbf{q}, \quad \Pi_{0}=\boldsymbol{\pi}, \\
\Pi_{1, k}^{j}=\exp \left(-\Gamma J\left(Q_{k}^{j}\right) h / 2\right) \Pi_{k}^{j}+\sigma\left(h / 2 ; Q_{k}^{j}\right) \eta_{k}^{j}, \\
j=1, \ldots, n, \\
R_{k}=-\frac{h}{2} \frac{\nu}{m} \nabla_{\mathbf{r}} U\left(\mathbf{R}_{k}, \mathbf{Q}_{k}\right)+\frac{\sqrt{h}}{2} \sqrt{\frac{2 \nu}{m \beta}} \xi_{k}, \\
\mathcal{R}_{k}=\mathbf{R}_{k}+2 \times \Delta R_{k}, \\
\Pi_{2, k}^{j}=\Pi_{1, k}^{j}-\frac{h}{2} \nabla_{q^{j}} U\left(\mathbf{R}_{k}, \mathbf{Q}_{k}\right), j=1, \ldots, n, \\
\left(\mathcal{Q}_{1, k}^{j}, \Pi_{3, k}^{j}\right)=\Psi_{3}\left(h / 2 ; Q_{k}^{j}, \Pi_{2, k}^{j}\right), \\
\left(\mathcal{Q}_{2, k}^{j}, \Pi_{4, k}^{j}\right)=\Psi_{2}\left(h / 2 ; \mathcal{Q}_{1, k}^{j}, \Pi_{3, k}^{j}\right) \\
\left(\mathcal{Q}_{3, k}^{j}, \Pi_{5, k}^{j}\right)=\Psi_{1}\left(h ; \mathcal{Q}_{2, k}^{j}, \Pi_{4, k}^{j}\right), \\
\left(\mathcal{Q}_{4, k}^{j}, \Pi_{6, k}^{j}\right)=\Psi_{2}\left(h / 2 ; \mathcal{Q}_{3, k}^{j}, \Pi_{5, k}^{j}\right) \\
\left(Q_{k+1}^{j}, \Pi_{7, k}^{j}\right)=\Psi_{3}\left(h / 2 ; \mathcal{Q}_{4, k}^{j}, \Pi_{6, k}^{j}\right), \quad j=1, \ldots, n,
\end{gathered}
$$

We are interested here in systems solutions of which satisfy a stronger condition, namely they are exponentially ergodic, i.e., for any $x \in \mathbb{R}^{14 n}$ and any function $\varphi$ with a polynomial growth:

$$
\left|E \varphi(X(t ; x))-\varphi^{e r g}\right| \leq C e^{-\lambda t}, \quad t \geq 0,
$$

where $C>0$ and $\lambda>0$ are some constants. In Refs. 8, 16, 18 (see also references therein), one can find conditions under which Langevin equations are exponentially ergodic.

It follows from (36) (and (35)) that for any $\varepsilon>0$ there exists $T_{0}>0$ such that for all $T \geq T_{0}$

$$
\left|E \varphi(X(T ; x))-\varphi^{e r g}\right| \leq \varepsilon .
$$

Then we can use the following estimate for the ergodic limit $\varphi^{\text {erg }}$ :

$$
\begin{aligned}
\varphi^{e r g} & \approx E \varphi(X(T ; x)) \approx E \varphi(\bar{X}(T ; x)) \\
& \approx \hat{\varphi}^{e r g}:=\frac{1}{L} \sum_{l=1}^{L} \varphi\left(\bar{X}^{(l)}(T ; x)\right),
\end{aligned}
$$


where $T$ is a sufficiently large time, $\bar{X}$ is an approximation of $X$, and $L$ is the number of independent approximate realizations. The total error

$$
R_{\hat{\varphi}^{e r g}}:=\hat{\varphi}^{e r g}-\varphi^{e r g}
$$

consists of three parts: the error $\varepsilon$ of the approximation $\varphi^{e r g}$ by $E \varphi(X(T ; x))$; the error of numerical integration $C h^{p}$ (see (21)), and the Monte Carlo error; i.e.,

$$
R_{\hat{\varphi}^{e r g}} \sim C h^{p}+\varepsilon+O\left(\frac{1}{\sqrt{L}}\right),
$$

or more specifically

$$
\begin{gathered}
\operatorname{Bias}\left(\hat{\varphi}^{e r g}\right)=\left|E \hat{\varphi}^{e r g}-\varphi^{e r g}\right| \leq C h^{p}+\varepsilon \\
\operatorname{Var}\left(\hat{\varphi}^{e r g}\right)=O(1 / L) .
\end{gathered}
$$

Each error is controlled by its own parameter: sufficiently large $T$ ensures smallness of the error $\mid \varphi^{\text {erg }}$ $E \varphi(X(T ; x)) \mid$; time step $h$ (as well as the choice of numerical method) controls the numerical integration error; the statistical error is regulated by choosing an appropriate number of independent trajectories $L$.

The other, commonly used in molecular dynamics, numerical approach to calculating ergodic limits is based on the known equality

$$
\lim _{t \rightarrow \infty} \frac{1}{t} \int_{0}^{t} \varphi(X(s ; x)) d s=\varphi^{e r g} \quad \text { a.s. }
$$

where the limit does not depend on $x$. Then by approximating a single trajectory, one gets the following estimator for $\varphi^{\text {erg }}$ :

$$
\varphi^{e r g} \sim \frac{1}{\tilde{T}} \int_{0}^{\tilde{T}} \varphi(X(s ; x)) d s \sim \check{\varphi}^{e r g}:=\frac{1}{L} \sum_{l=1}^{L} \varphi(\bar{X}(l h ; x)),
$$

where $\tilde{T}$ is sufficiently large and $L h=\tilde{T}$. In Ref. 17 this approach was rigorously justified in the case of ergodic SDEs with nondegenerate noise and globally Lipschitz coefficients. Let us emphasize that $\tilde{T}$ in (41) is much larger than $T$ in (37) and (38) because $\tilde{T}$ should be such that it not just ensures the distribution of $X(t)$ to be close to the invariant distribution (like it is required from $T$ ) but it should also guarantee smallness of variance of $\check{\varphi}^{\text {erg }}$. See further details concerning computing ergodic limits in Ref. 12 and references therein.

\section{NUMERICAL INVESTIGATION}

In this section we present a numerical study of the Langevin and gradient-Langevin thermostats derived in Section [II In particular, we investigate the dependence of the thermostat properties on the choice of parameters $\gamma$ and $\Gamma$ for the Langevin system (14)-(15) and $\nu$ and $\Gamma$ for the gradient-Langevin system (17)-(18), as well as the dependence of the numerical discretization errors of the numerical schemes Langevin A, Langevin B, and gradientLangevin on the integration step size $h$. As a model system, we use the popular TIP4P rigid model of water ${ }^{19}$. In order to speed up the simulations, both Lennard-Jones and electrostatic interactions are smoothly turned off between 9.5 and $10 \AA$. This truncation has minimal effect on the structure of liquid water, but leads to a lower estimated melting temperature ${ }^{20}$ of $219 \mathrm{~K}$.

The two key requirements of a thermostat are: i) correct sampling of phase space points distributed according to the Gibbs distribution at a desired thermostat temperature $T$, and ii) rapid relaxation of the system to the desired equilibrium state. The numerical accuracy of the sampling can be estimated by comparing the values of various system properties (e.g. kinetic and potential energies, pressure) averaged over long simulation runs to those obtained with a much smaller step size $h$.

To estimate how quickly the system relaxes to the desired equilibrium state we use the following simple experiment. A system of 2000 TIP4P water molecules is equilibrated at $T_{0}=220 \mathrm{~K}$. Then the temperature of the thermostat is increased (instantaneously) to $T_{1}=270 \mathrm{~K}$, and the run is continued until the system is equilibrated at the new temperature. We deliberately choose to simulate the system at lower temperatures (close to the melting temperature for this model of water), where the relaxation of the system is expected to be slower.

Assuming that the system is exponentially ergodic (see (36)), we can expect that any measured quantity $A$ will relax from its equilibrium value $A_{0}$ at $T_{0}$ to the equilibrium value $A_{1}$ at $T_{1}$ according to the approximate formula

$$
E A(t) \equiv\langle A(t)\rangle \doteq A_{1}+\left(A_{0}-A_{1}\right) \exp \left(-t / \tau_{A}\right),
$$

where $\tau_{A}$ is the characteristic relaxation time of the quantity $A$. The temperature switch occurs at $t=0$ and the angle brackets denote average over an ensemble of independent simulation runs. The subscript on $\tau_{A}$ indicates that different quantities may relax with different rates. The rate of system equilibration should be estimated from the maximum value of $\tau_{A}$ among the quantities of interest.

The quantities we measure include the translational kinetic temperature

$$
\mathcal{T}_{\text {tr }}=\frac{\mathbf{p}^{\top} \mathbf{p}}{3 n k_{B} m}
$$

rotational kinetic temperature

$$
\mathcal{T}_{\text {rot }}=\frac{2}{3 n k_{B}} \sum_{j=1}^{n} \sum_{l=1}^{3} V_{l}\left(q^{j}, \pi^{j}\right),
$$

and potential energy per molecule

$$
\mathcal{U}=\frac{1}{n} U(\mathbf{r}, \mathbf{q}) .
$$




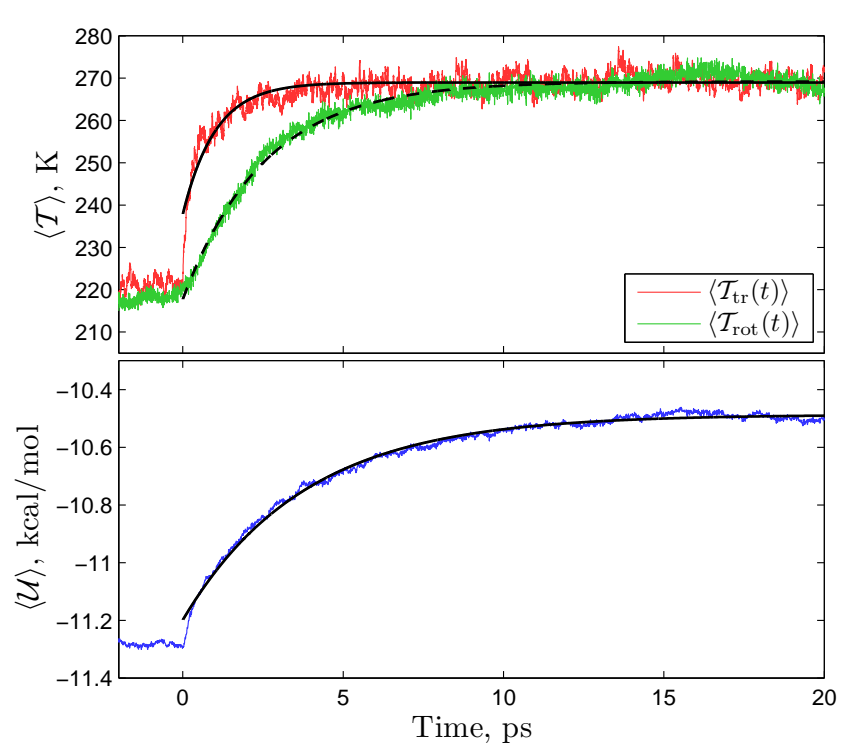

FIG. 1: (Color online) Relaxation dynamics with translational Langevin thermostat: $\gamma=4.0 \mathrm{ps}^{-1}, \Gamma=0$. Thin lines show relaxation dynamics averaged over ten independent runs, thick lines (solid and dashed for translational and rotational temperatures, respectively) show the least squares fit to formula (42). The estimated values of the relaxation times are $\tau_{\mathcal{T}_{\text {tr }}}=0.2 \mathrm{ps}, \tau_{\mathcal{T}_{\text {rot }}}=1.9 \mathrm{ps}$, and $\tau_{\mathcal{U}}=3.6 \mathrm{ps}$.

To illustrate the response of the system to the instantaneous temperature change, we show in Fig. 1 the result of applying the Langevin thermostat only to the translational degrees of freedom, i.e. $\Gamma=0$ in (14)-(15). As expected, the translational kinetic temperature quickly relaxes to the new temperature, while the rotational kinetic temperature and potential energy lag behind. To estimate the relaxation rates of the measured quantities, we use the least squares fit of the exponential function (42) to the average measured quantity $\langle A(t)\rangle$.

In all the simulations we performed, the potential energy relaxation time is larger than that for either of the kinetic temperatures. Therefore, we determine the relaxation time of the system to the new equilibrium state based on the value of $\tau_{\mathcal{U}}$.

Varying the value of the translational Langevin parameter $\gamma$, we observe that relaxation is slower for both small and large values of $\gamma$, with the fastest relaxation around $\gamma=4.0 \mathrm{ps}^{-1}$. The existence of an optimal value for the choice of the thermostat parameter is consistent with observations in Ref. 15 and can be understood in terms of the interaction of the system with the thermostat. For small values of $\gamma$, the relaxation of the system is slow due to the limited heat flux between the system and the thermostat. For large $\gamma$, even though the kinetic temperature relaxes very quickly, the relaxation of the configurational state of the system is apparently hindered by the disruptive influence of the random force on the Hamiltonian dynamics which is driving the system to the new equilibrium.

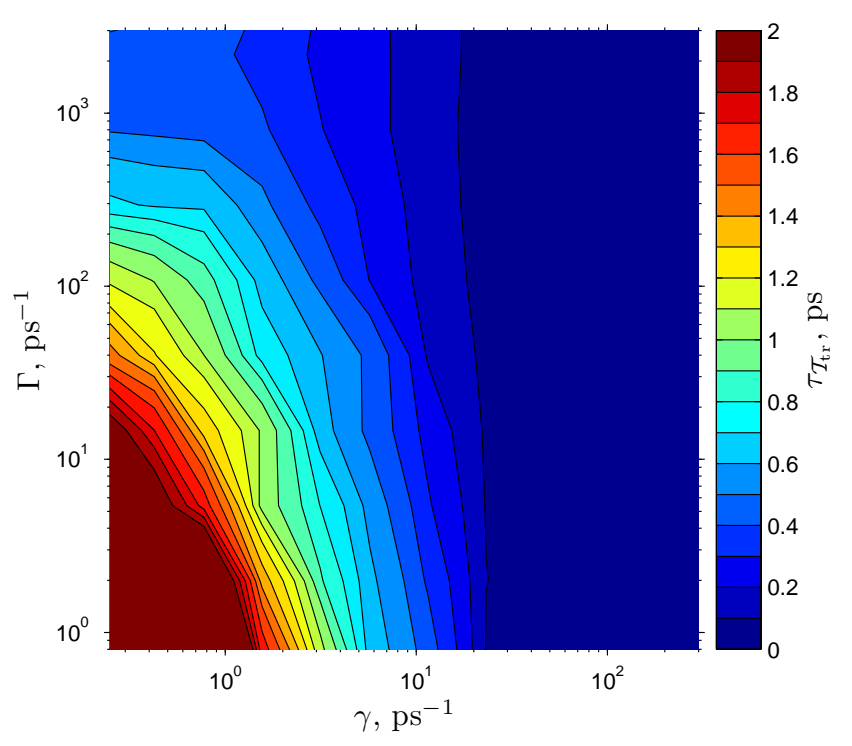

FIG. 2: (Color online) Translational temperature relaxation time for the Langevin thermostat (14)-(15) as a function of the thermostat parameters $\gamma$ and $\Gamma$.

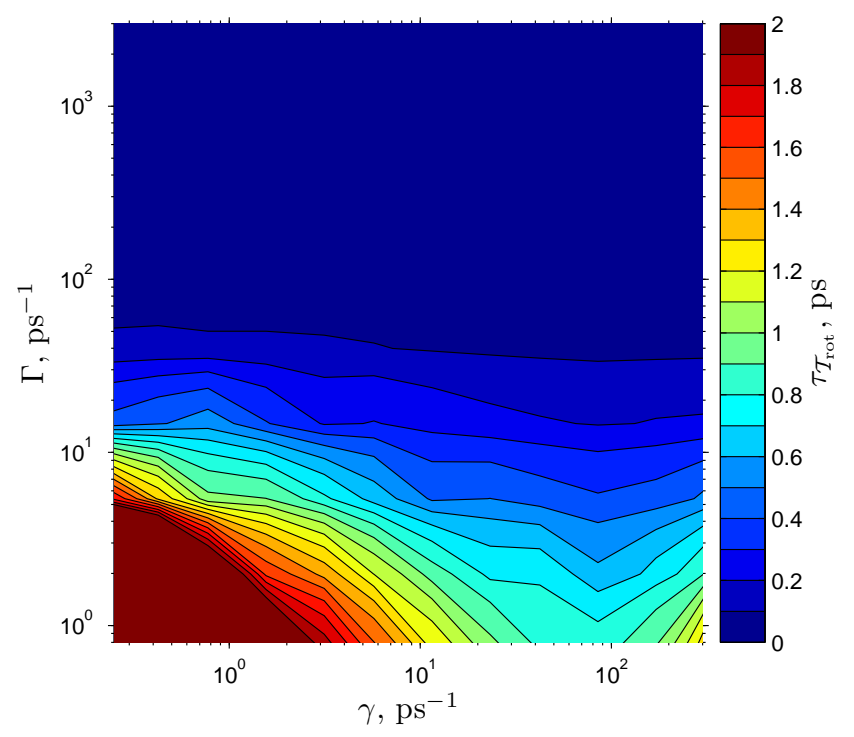

FIG. 3: (Color online) Rotational temperature relaxation time for the Langevin thermostat (14)-115) as a function of the thermostat parameters $\gamma$ and $\Gamma$.

\section{A. Langevin Thermostats}

Next, we investigate the dependence of the system relaxation time $\tau_{\mathcal{U}}$ on both $\gamma$ and $\Gamma$ in (14)-(15). In order to minimize the influence of the numerical discretization error, we use a relatively small time step of $0.2 \mathrm{fs}$. With such a small time step, the difference between Langevin A and Langevin B is negligible compared to the sampling error. To produce the results reported below, we use Langevin A. We evaluate $\tau_{\mathcal{U}}$ on a logarithmic grid of $\gamma$ and $\Gamma$ values using five independent runs at each 


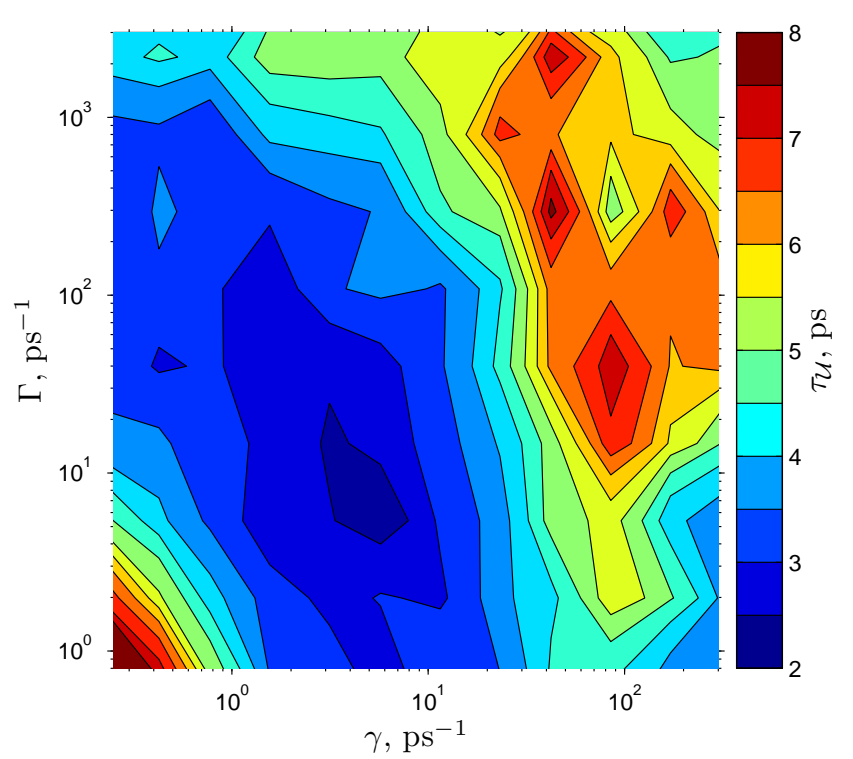

FIG. 4: (Color online) Potential energy relaxation time for the Langevin thermostat (14)-(15) as a function of the thermostat parameters $\gamma$ and $\Gamma$.

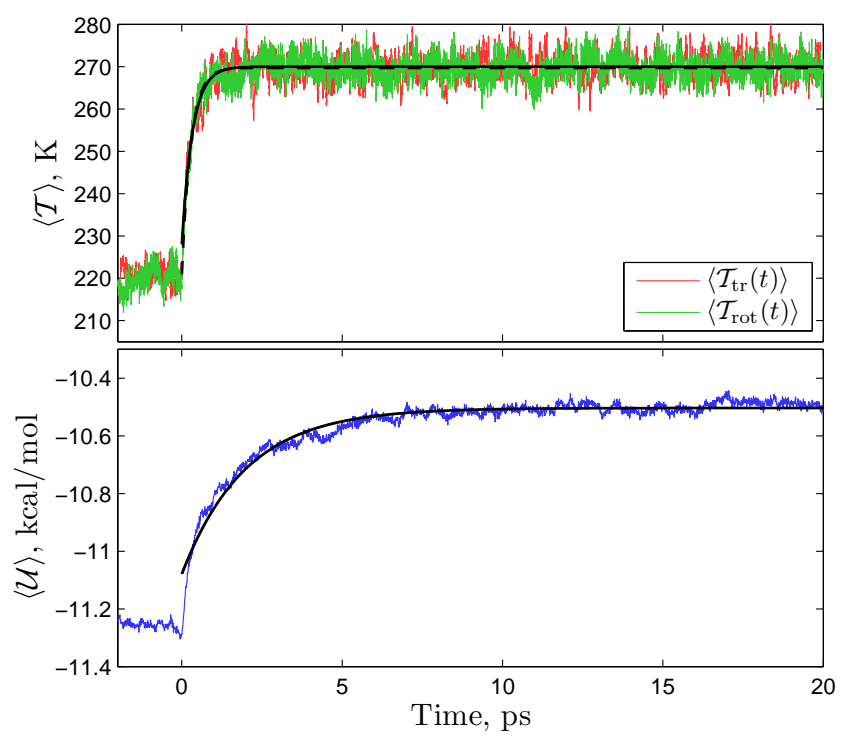

FIG. 5: (Color online) Relaxation dynamics with 'optimal' choice of Langevin thermostat parameters: $\gamma=4.0 \mathrm{ps}^{-1}$, $\Gamma=10.0 \mathrm{ps}^{-1}$. Thin lines show relaxation dynamics averaged over ten independent runs, thick lines (solid and dashed for translational and rotational temperatures, respectively) show the least squares fit to formula (42). The estimated values of the relaxation times are $\tau_{\mathcal{T}_{\text {tr }}}=0.28 \mathrm{ps}, \tau_{\mathcal{T}_{\text {rot }}}=0.26 \mathrm{ps}$, and $\tau_{\mathcal{U}}=2.0 \mathrm{ps}$.

point. The results are shown in Figs. 2, 3, and 4, As expected, the relaxation speed of translational and rotational temperatures uniformly increases with increasing values of $\gamma$ and $\Gamma$, respectively. At the same time, the relaxation speed of the potential energy exhibits nonuniform dependence on the thermostat parameters. As can

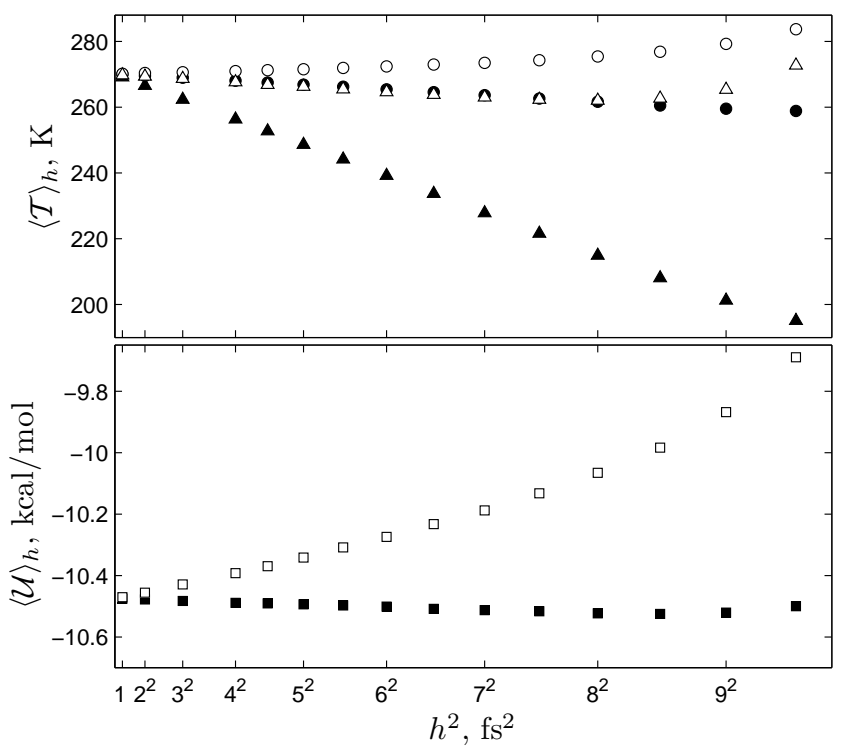

FIG. 6: Dependence of the approximated average properties of a system of 2000 TIP4P water molecules on the integration time step $h$ for Langevin A and B. The system is equilibrated with the thermostat parameters $\gamma=4.0 \mathrm{ps}^{-1}, \Gamma=10.0 \mathrm{ps}^{-1}$, and $T=270 \mathrm{~K}$. The quantities $\left\langle\mathcal{T}_{\text {tr }}\right\rangle_{h},\left\langle\mathcal{T}_{\text {rot }}\right\rangle_{h}$, and $\langle\mathcal{U}\rangle_{h}$ are denoted by circles, triangles, and squares, respectively. Solid and open symbols refer to Langevin A and B, respectively.

be seen in Fig. 4, the fastest relaxation of the system is achieved when the Langevin thermostat is applied to both translational and rotational degrees of freedom, with $\gamma=2-8 \mathrm{ps}^{-1}$ and $\Gamma=3-40 \mathrm{ps}^{-1}$. The relaxation dynamics of the system with 'optimal' choice of Langevin thermostat parameters is demonstrated in Fig. 5. In this case $\tau_{\mathcal{T}_{\text {tr }}}=0.28 \mathrm{ps}, \tau_{\mathcal{T}_{\text {rot }}}=0.26 \mathrm{ps}$, and $\tau_{\mathcal{U}}=2.0 \mathrm{ps}$, which shows that the system relaxation is almost twice as fast as when the Langevin thermostat is applied only to translational degrees of freedom. Note that the results shown in Fig. [4 for $\gamma$ larger than about $100 \mathrm{ps}^{-1}$ or $\Gamma$ larger than about $1000 \mathrm{ps}^{-1}$ are not reliable due to excessive coupling of the system to the thermostat, which disrupts the Hamiltonian flow of the system. In this case, the relaxation dynamics is poorly represented by the exponential function (42) and thus the fits produce misleading values for the system relaxation time.

Now we look at performance of the numerical integrators proposed in Section $\amalg$ II A for the Langevin thermostat (14)-(15). Since both Langevin A and B are secondorder methods, the calculated average quantities for simulations with step size $h$ should have the form 11,21

$$
\langle A\rangle_{h}=\langle A\rangle_{0}+C_{A} h^{2}+O\left(h^{3}\right),
$$

where $\langle A\rangle_{h}$ denotes the average value of dynamical quantity $A(t)$ calculated over a numerical trajectory with time step $h$. The dependence of $\left\langle\mathcal{T}_{\text {tr }}\right\rangle_{h},\left\langle\mathcal{T}_{\text {rot }}\right\rangle_{h}$, and $\langle\mathcal{U}\rangle_{h}$ on $h$ for both Langevin A and B is illustrated in Fig. 6. It appears that Langevin A has larger discretization error for the rotational temperature and smaller error for the 
TABLE I: Values of the coefficients $C_{A}$ in the discretization errors (46) for the measured quantities with Langevin A and B. The system's parameters are as in Fig. 6 .

\begin{tabular}{l|cc}
\hline \hline & Langevin $\mathrm{A}$ & Langevin B \\
\hline$C_{\mathcal{T}_{\text {tr }}}, \mathrm{K} / \mathrm{fs}^{2}$ & -0.13 & 0.06 \\
$C_{\mathcal{T}_{\text {rot }}}, \mathrm{K} / \mathrm{fs}^{2}$ & -0.85 & -0.14 \\
$C_{\mathcal{U}}, \mathrm{kcal} / \mathrm{mol} / \mathrm{fs}^{2}$ & -0.0007 & 0.0056 \\
\hline \hline
\end{tabular}

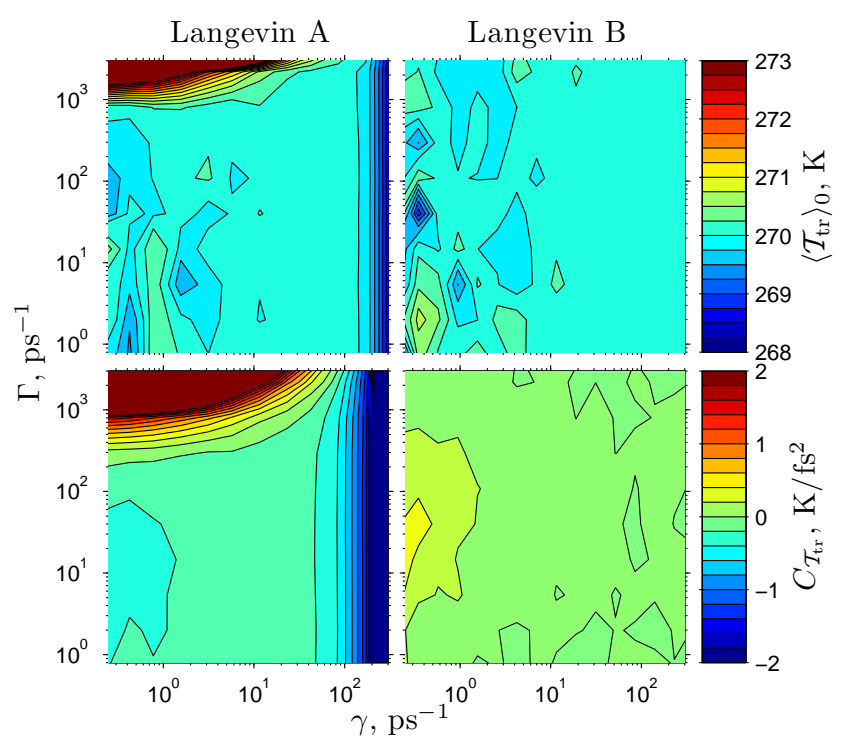

FIG. 7: (Color online) Dependence of $\left\langle\mathcal{T}_{\mathrm{tr}}\right\rangle_{0}$ and $C_{\mathcal{T}_{\text {tr }}}$ on $\gamma$ and $\Gamma$ for Langevin $\mathrm{A}$ and $\mathrm{B}$ thermostats.

potential energy than Langevin B. The linear dependence of the measured quantities on $h^{2}$ is maintained up to a relatively large time step of about $h=7 \mathrm{fs}$. The values of the slopes $C_{A}$ are listed in Table I. Both methods become unstable at about $h=10 \mathrm{fs}$.

We have investigated the dependence of $\langle A\rangle_{0}$ and $C_{A}$ on the thermostat parameters $\gamma$ and $\Gamma$, by running simulations with time steps $h=2$ fs and 3 fs and estimating these quantities from the straight line fit with respect to $h^{2}$. We consider the fit to be justified if the higher order terms in (46) are small, i.e., when the quantities $\left\langle\mathcal{T}_{\text {tr }}\right\rangle_{0}$ and $\left\langle\mathcal{T}_{\text {rot }}\right\rangle_{0}$ determined from (46) are equal to the thermostat temperature parameter $T=270 \mathrm{~K}$. In Fig. 7 we show results for the translational temperature measurements in simulations with Langevin A and B. As expected, $\left\langle\mathcal{T}_{\text {tr }}\right\rangle_{0}$ converges to $270 \mathrm{~K}$. We note in passing that smaller statistical errors are observed at larger values of $\gamma$. The behavior of $C_{\mathcal{T}_{\text {tr }}}$ for Langevin A exhibits a plateau for small and moderate values of $\gamma$ and $\Gamma$ and then changes rapidly at values that are 'too large' for this system. By contrast, the translational temperature discretization error of Langevin B thermostat exhibits consistent behavior for all values of $\gamma$ and $\Gamma$. Similar differences between Langevin A and B can be seen in the measurements of rotational temperature and poten-

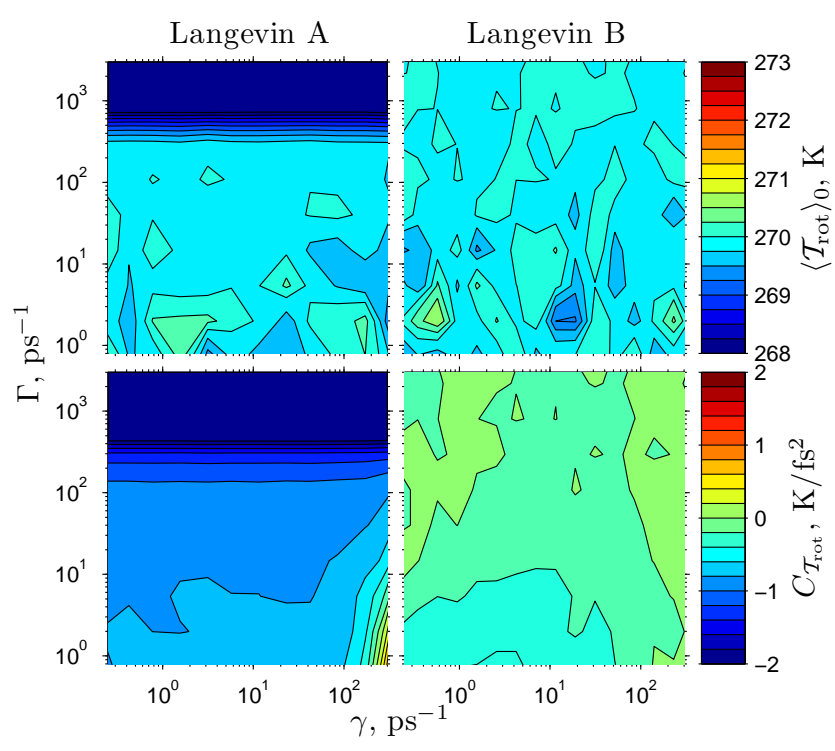

FIG. 8: (Color online) Dependence of $\left\langle\mathcal{T}_{\text {rot }}\right\rangle_{0}$ and $C_{\mathcal{T}_{\text {rot }}}$ on $\gamma$ and $\Gamma$ for Langevin A and B thermostats.

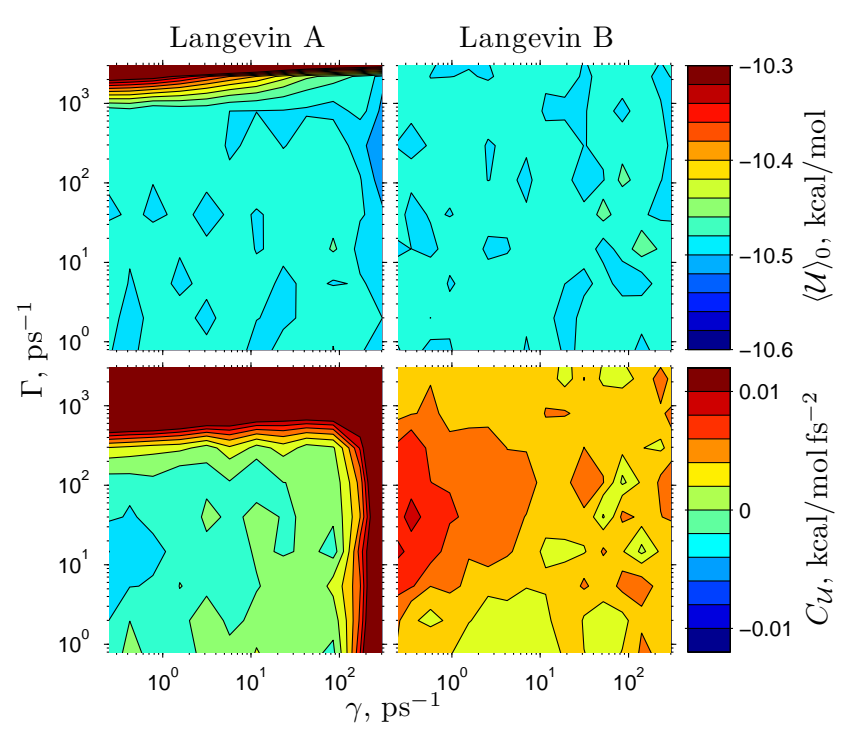

FIG. 9: (Color online) Dependence of $\langle\mathcal{U}\rangle_{0}$ and $C \mathcal{U}$ on $\gamma$ and $\Gamma$ for Langevin A and B thermostats.

tial energy shown in Figs. 8 and 9, respectively. As can be seen from the plot of $\left\langle\mathcal{T}_{\text {rot }}\right\rangle_{0}$ in Fig. 8 , the straight line fit also breaks down at large $\Gamma$ values in Langevin A.

\section{B. Gradient-Langevin thermostat}

Here we describe numerical experiments with the gradient-Langevin thermostat (17)-(18) introduced in Section [IB. Since the gradient system for the translational motion does not include linear momenta $\mathbf{P}$, the translational kinetic temperature $\mathcal{T}_{\text {tr }}$ is not available for measurement in this case. Therefore, in our numerical 


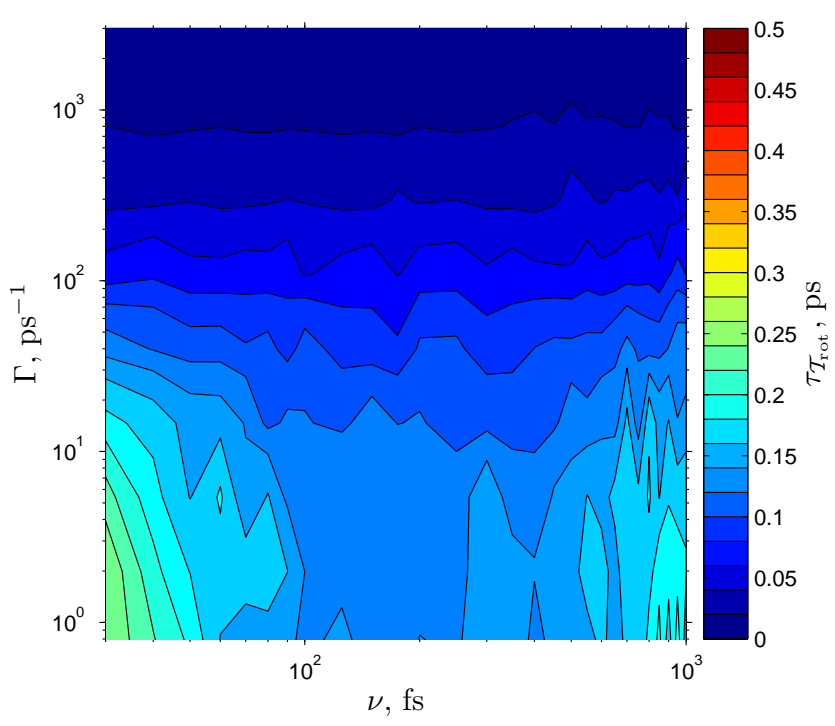

FIG. 10: (Color online) Rotational temperature relaxation time for the gradient-Langevin thermostat (17)-(18) as a function of the thermostat parameters $\nu$ and $\Gamma$.

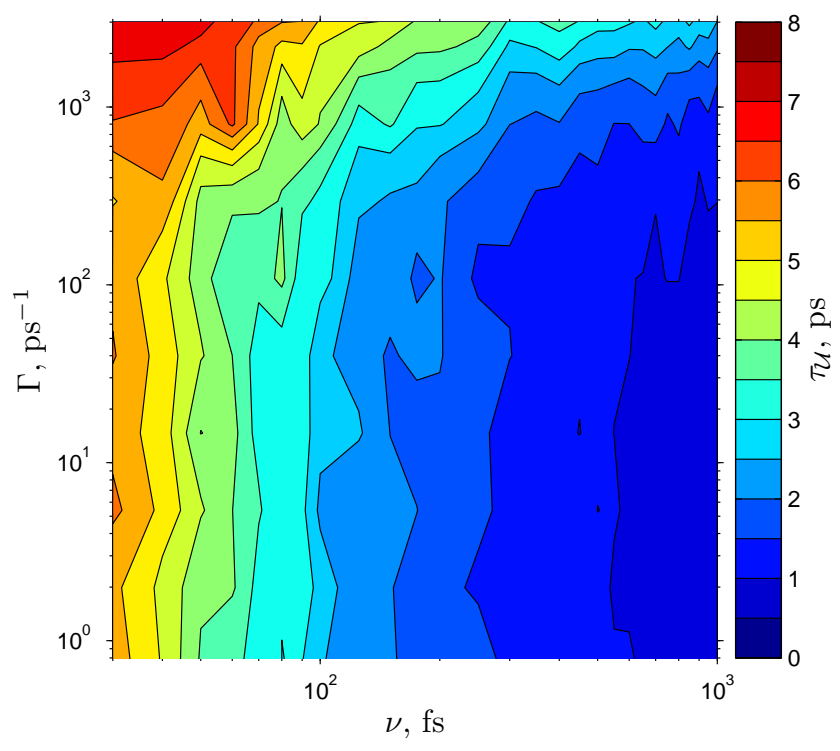

FIG. 11: (Color online) Potential energy relaxation time for the gradient-Langevin thermostat (17)-(18) as a function of the thermostat parameters $\nu$ and $\Gamma$.

experiments we measure the rotational temperature $\mathcal{T}_{\text {rot }}$ and potential energy per particle $\mathcal{U}$ as defined by (44) and (45), respectively.

For the particular system studied here, the gradientLangevin numerical scheme (34), (25) from Section IIIB becomes unstable when the product $h \nu$ is larger than about $200 \mathrm{fs}^{2}$. Therefore, with the step size of $h=0.2 \mathrm{fs}$ used in our simulations, we can study the properties of the gradient-Langevin scheme with $\nu$ up to about $1000 \mathrm{fs}$.

We conducted the relaxation experiment, where we monitored $\left\langle\mathcal{T}_{\text {rot }}(t)\right\rangle$ and $\langle\mathcal{U}(t)\rangle$ while the thermostat tem-

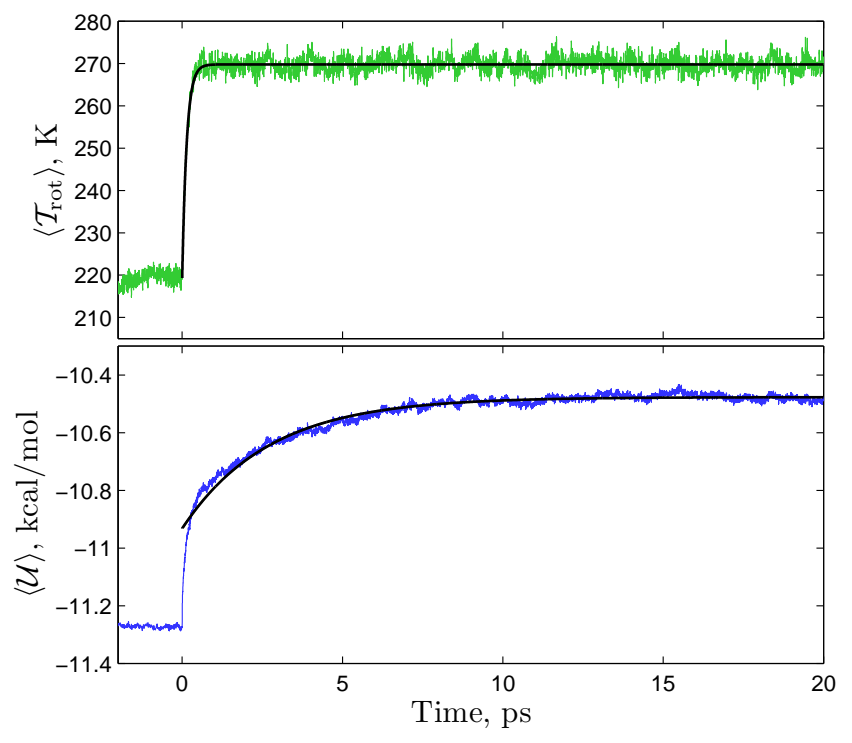

FIG. 12: (Color online) Relaxation dynamics with gradientLangevin thermostat: $\nu=100 \mathrm{fs}, \Gamma=0$. Thin lines show relaxation dynamics averaged over seven independent runs, thick lines show the least squares fit to formula (42).

perature parameter was switched from $220 \mathrm{~K}$ to $270 \mathrm{~K}$. The relaxation times $\tau_{\mathcal{T}_{\text {rot }}}$ and $\tau_{\mathcal{U}}$ for these quantities were calculated for different values of $\nu$ and $\Gamma$. The results are shown in Figs. 10 and 11. As expected, the relaxation time for rotational temperature decreases with increasing value of $\Gamma$. The somewhat surprising finding of this experiment is that, even for small values of $\Gamma$ the relaxation time is much smaller here than in the case of Langevin thermostat (see Fig. 3). As an illustration, we show the relaxation experiment for $\nu=100 \mathrm{fs}$ and $\Gamma=0$ in Fig. 12, The estimated relaxation time for rotational temperature, $\tau_{\mathcal{T}_{\text {rot }}}=0.14 \mathrm{ps}$, is much smaller then the corresponding quantity for the Langevin thermostat, even though the relaxation time for the potential energy, $\tau_{\mathcal{U}}=2.7 \mathrm{ps}$, is similar. This indicates a very efficient heat transfer between the gradient dynamics of the translational motion and the rotational motion.

The dependence of $\tau_{\mathcal{U}}$ on the thermostat parameters for the gradient-Langevin system shown in Fig. 11 is markedly different than for the Langevin system. In particular, the relaxation time decreases with increasing $\nu$ without reaching a minimum value within the range of $\nu$ values explored. At the same time, there is little dependence on $\Gamma$, except for very large $\Gamma$ where, similar to the Langevin system, the measurements of the relaxation time are not reliable. Also, note that the relaxation times are much smaller, reaching as low as $0.7 \mathrm{ps}$ for $\nu=1000 \mathrm{fs}$, compared to the minimum value of about $2.0 \mathrm{ps}$ for the Langevin system.

Of course, the direct comparison between the relaxation speeds of gradient-Langevin and Langevin dynamics has to be taken with caution, since, as we mentioned in Section IIB, the gradient dynamics does not have a 


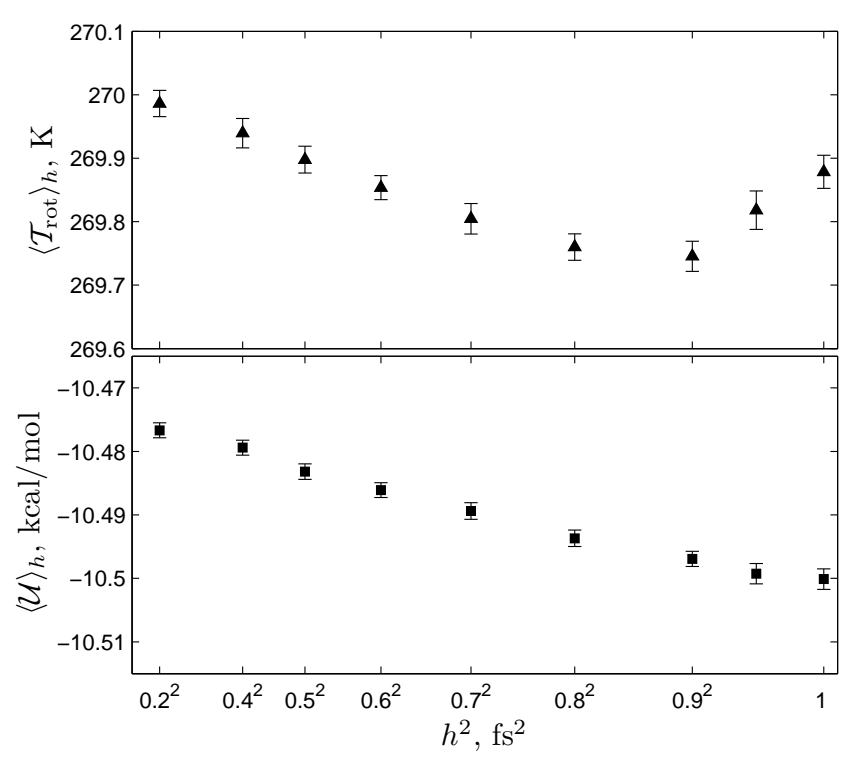

FIG. 13: Dependence of the approximated average properties of a system of 2000 TIP4P water molecules on the integration time step $h$ for the gradient-Langevin numerical method. The system is equilibrated with the thermostat parameters $\nu=$ $200 \mathrm{fs}, \Gamma=5.0 \mathrm{ps}^{-1}$, and $T=270 \mathrm{~K}$. The quantities $\left\langle\mathcal{T}_{\text {rot }}\right\rangle_{h}$, and $\langle\mathcal{U}\rangle_{h}$ are denoted by circles and squares, respectively. Error bars reflect $95 \%$ confidence intervals in the obtained results estimated from block averages.

natural evolution time. In particular, the mass of the molecule $m$ does not have a specific meaning in the gradient system, since it can be rescaled to any value together with $h$ and $\nu$ (see (17)). Computationally, the relaxation speed depends on the time step $h$ and, while with $\nu=1000 \mathrm{fs}$ the gradient Langevin scheme becomes unstable for $h$ larger than $0.2 \mathrm{fs}$, the Langevin scheme remains stable up to about $h=10$ fs for the optimal values of $\gamma=4.0 \mathrm{ps}^{-1}$ and $\Gamma=10 \mathrm{ps}^{-1}$.

The dependence of discretization error in measured quantities on $h$ for the gradient-Langevin scheme is shown in Fig. 13. As in the case of Langevin A and B, we clearly see the linear dependence of $\left\langle\mathcal{T}_{\text {rot }}\right\rangle_{h}$, and $\langle\mathcal{U}\rangle_{h}$ on $h^{2}$. The estimated slopes in (46) are $C_{\mathcal{T}_{\text {rot }}}=-0.38 \mathrm{~K} / \mathrm{fs}^{2}$ and $C_{\mathcal{U}}=-0.029 \mathrm{kcal} / \mathrm{mol} / \mathrm{fs}^{2}$. Unfortunately, for this value of $\nu$ the gradient-Langevin numerical integrator becomes unstable for $h>1 \mathrm{fs}$, which is rather small, given that the Langevin A and B integrator are stable for $h$ up to about $10 \mathrm{fs}$. Still, given the observed efficient heat transfer from the gradient subsystem for translational dynamics to the rotational dynamics (see Fig. 12 and related discussion), it might be of interest to construct numerical methods for the gradient-Langevin system (17)(18) with better stability properties than those of (34),
(25); this has not been considered in this paper.

\section{SUMMARY}

The new stochastic thermostats presented in this paper are appropriate for quaternion-based rigid body models. They are written in the form of Langevin equations and gradient-Langevin system (gradient subsystem for the translational degrees of freedom and Langevin subsystem for the rotational degrees of freedom). The obtained stochastic systems preserve the unit length of the rotational coordinates in the quaternion representation of the rigid-body dynamics. The thermostats allow to couple both translational and rotational degrees of freedom to the "heat bath". As it is shown in the numerical tests with the TIP4P rigid model of water, the Langevin thermostat relaxes to an equilibrium faster when not only translational degrees of freedom but also rotational ones are thermostated. It turns out that there is an optimal range of the strength of coupling to the "heat bath". In contrast, the gradient-Langevin thermostat has a monotone dependence of relaxation time on the thermostat parameters. In the case of the Langevin thermostat, two quasi-symplectic second-order (in the weak sense) integrators are constructed and compared in the numerical tests. For the gradient-Langevin thermostat, a RungeKutta second-order method is proposed. All the methods preserve the unit length of the rotational coordinates. The numerical experiments demonstrate the efficiency of the proposed thermostating technique.

Relaxation times for the gradient-Langevin thermostat are smaller than for the Langevin thermostat. However, the numerical methods proposed for the Langevin system have better stability properties than the scheme used for numerical integration of the gradient-Langevin system. In our experimental study, the use of the Langevin thermostat together with the quasi-symplectic integrators was computationally significantly more efficient than thermostating via the gradient-Langevin system and the numerical scheme for it.

\section{Acknowledgments}

The work of RLD and RH was supported by the EPSRC research grant GR/T27105/01 which is gratefully acknowledged. One of the authors (RLD) did part of the work during his study leave granted by the University of Leicester. The computations were performed on the University of Leicester Mathematical Modelling Centres cluster, which was purchased through the EPSRC strategic equipment initiative.

${ }^{1}$ Hünenberger, P.H., Thermostat algorithms for molecular 
${ }^{2}$ Miller III, T.F., Eleftheriou, M., Pattnaik, P., Ndirango, A., Newns, D., Matyna, G.J., Symplectic quaternion scheme for biophysical molecular dynamics, J. Chem. Phys., 116 (2002), 8649-8659.

3 Leimkuhler, B., Reich, S., Simulating Hamiltonian Dynamics, Cambridge Univ. Press, 2005.

4 Vanden-Eijnden, E., Ciccotti, G., Second-order integrators for Langevin equations with holonomic constraints, Chem. Phys. Lett., 429 (2006), 310-316.

5 Sun, X., Lin, T., Gezelter, J. D., Langevin dynamics for rigid bodies of arbitrary shape, J. Chem. Phys., 128 (2008), 234107.

6 Izaguirre, J.A., Catarello, D.P., Wozniak, J.M., Skeel, R.D. Langevin stabilization of molecular dynamics, J. Chem. Phys., 114 (2001), 2090-2098.

7 Hasminskii, R.Z., Stochastic Stability of Differential Equations, Sijthoff \& Noordhoff, 1980.

8 Mattingly, J.C., Stuart, A.M., Higham, D.J., Ergodicity for SDEs and approximations: locally Lipschitz vector fields and degenerate noise, Stoch. Proc. Appl., 101 (2002), 185-232.

9 Milstein, G.N., Repin, Yu.M., Tretyakov, M.V., Symplectic integration of Hamiltonian systems with additive noise, SIAM J. Numer. Anal., 39 (2002), 2066-2088.

10 Milstein, G.N., Tretyakov, M.V., Quasi-symplectic methods for Langevin-type equations, IMA J. Numer. Anal., 23 (2003), 593-626.

11 Milstein, G.N., Tretyakov, M.V., Stochastic Numerics for Mathematical Physics, Springer, 2004.

12 Milstein, G.N., Tretyakov, M.V., Computing ergodic limits for Langevin equations, Physica D, 229 (2007), 81-95.

13 Nelson, E., Dynamical Theories of Brownian Motion,
Princeton Univ. Press, 1967.

14 Skeel, R., Integration schemes for molecular dynamics and related applications, In: "Graduate Student's Guide to $\mathrm{Nu}$ merical Analysis'98" edited by M. Ainsworth, J. Levesley, M. Marletta, Springer, 1999, 118-176.

15 Bussi, G., Parrinello, M., Accurate sampling using Langevin dynamics, Phys. Rev. E 75 (2007), 056707.

16 Soize, C., The Fokker-Planck Equation for Stochastic Dynamical Systems and its Explicit Steady State Solutions, World Scientific, 1994.

17 Talay, D., Second-order discretization schemes for stochastic differential systems for the computation of the invariant law, Stoch. Stoch. Rep., 29 (1990), 13-36.

18 Talay, D., Stochastic Hamiltonian systems: exponential convergence to the invariant measure, and discretization by the implicit Euler scheme, Markov Proc. Relat. Fields, 8 (2002), 163-198.

19 Jorgensen, W.L., Chandrasekhar, J., Madura, J., Impey, R.W., Klein, M.L., Comparison of simple potential functions for simulating liquid water, J. Chem. Phys., 79 (1983), 926-935.

20 Handel, R., Davidchack, R.L., Anwar, J., Brukhno, A., Direct calculation of solid-liquid interfacial free energy for molecular systems: TIP4P ice-water interface, Phys. Rev. Lett., 100 (2008), 036104.

21 Talay, D., Tubaro, L., Expansion of the global error for numerical schemes solving stochastic differential equations, Stoch. Anal. Appl., 8 (1990), 483-509.

22 Following the standard notation of the SDE theory, we use capital letters to denote the SDE solution and small letters for the initial data and for corresponding "dummy" variables. 